\title{
ON CW-COMPLEXES OVER GROUPS WITH PERIODIC COHOMOLOGY
}

\author{
JOHN NICHOLSON
}

\begin{abstract}
If $G$ has 4-periodic cohomology, then finite D2 complexes over $G$ are determined up to polarised homotopy by their Euler characteristic if and only if $G$ has at most two one-dimensional quaternionic representations. We use this to solve Wall's D2 problem for several infinite families of non-abelian groups and, in these cases, also show that any finite Poincaré 3-complex $X$ with $G=\pi_{1}(X)$ admits a cell structure with a single 3-cell. The proof involves cancellation theorems for $\mathbb{Z} G$ modules where $G$ has periodic cohomology.
\end{abstract}

\section{INTRODUCTION}

In the $1960 \mathrm{~s}, \mathrm{C}$. T. C. Wall defined a $\mathrm{D} n$ complex to be a CW-complex $K$ that $H_{i}(\widetilde{K})=0$ for $i>n$ and $H^{n+1}(K ; M)=0$ for all finitely generated $\mathbb{Z}\left[\pi_{1}(K)\right]$ modules $M$. Wall showed that a finite $\mathrm{D} n$ complex is homotopy equivalent to a finite $n$-complex provided $n>2$ Wa65. This was extended to the case $n=1$ by Stallings-Swan [St68, Sw69, but the question for $n=2$ remains completely open:

D2 problem. Is every finite D2 complex homotopy equivalent to a finite 2-complex?

We say that a finitely presented group $G$ has the $D 2$ property if every finite D2 complex $X$ with $\pi_{1}(X)=G$ is homotopy equivalent to a finite 2-complex.

In Wa67, Wall applied this to the question of whether a finite Poincaré $n$ complex $X$ could be given a cell structure with a single $n$-cell $e^{n}$. He showed that

$$
X=K \cup e^{n}
$$

where $K$ is a finite $\mathrm{D}(n-1)$-complex and so, if $K$ is homotopy equivalent to a finite $(n-1)$-complex, then $X=K \cup e^{n}$ gives a cell structure with a single $n$-cell. In particular, if $n \neq 3$, then every finite Poincaré $n$-complex admits a cell structure with a single $n$-cell. The case $n=3$ remains open though would be true provided the fundamental groups of finite Poincaré 3-complexes had the D2 property.

Since the finite fundamental groups of Poincaré 3-complexes have 4-periodic cohomology, the D2 property for such groups is of special interest. In fact, it was conjectured by J. M. Cohen that all other groups have the D2 property [Co77, p415]. In light of this, groups with 4-periodic cohomology have been the source of many proposed counterexamples [Co77, Jo04, BW05. The only cases previously known to have the D2 property are cyclic, dihedral, or binary polyhedral Dy75, Jo03a].

Our main result is the following partial classification of finite D2 complexes whose fundamental group has 4-periodic cohomology. Let $m_{\mathbb{H}}(G)$ denote the number of copies of $\mathbb{H}$ in the Wedderburn decomposition of $\mathbb{R} G$ for a finite group $G$.

Theorem A. Let $G$ have 4-periodic cohomology. Then finite D2 complexes $X$ with $\pi_{1}(X)=G$ are determined up to (polarised) homotopy by their Euler characteristic if and only if $m_{\mathbb{H}}(G) \leq 2$.

2020 Mathematics Subject Classification. Primary 57K20; Secondary 20C05, 57P10, 57Q12. 
Here we assume that each finite D2 complex $X$ comes equipped with an isomorphism $\rho_{X}: \pi_{1}(X) \cong G$ and, for pairs $\left(X, \rho_{X}\right)$ and $\left(Y, \rho_{Y}\right)$, we say that a homotopy equivalence $h: X \rightarrow Y$ is a polarised homotopy equivalence if $\rho_{Y} \circ \pi_{1}(h) \circ \rho_{X}^{-1}=\mathrm{id}_{G}$.

Recall that every finite 2-complex $X$ with $\pi_{1}(X)=G$ is homotopy equivalent to the Cayley complex $X_{\mathcal{P}}$ of some presentation $\mathcal{P}=\left\langle s_{1}, \cdots, s_{n} \mid r_{1}, \cdots, r_{m}\right\rangle$ of $G$, and that $\chi\left(X_{\mathcal{P}}\right)=1-\operatorname{def}(\mathcal{P})$ where $\operatorname{def}(\mathcal{P})=n-m$ is the deficiency of $\mathcal{P}$. If $G$ is finite, then $\operatorname{def}(\mathcal{P}) \geq 0$ (see, for example, [Sw65, Corollary 1.3]). In Theorem 4.1 we show that, for every group $G$ with 4-periodic cohomology, there exists a finite D2 complex $X$ for which $\pi_{1}(X)=G$ and $\chi(X)=1$. In particular, we will show:

Corollary B. Let $G$ have 4-periodic cohomology. Then:

(i) If $G$ has the D2 property, then $G$ has a balanced presentation.

(ii) If $G$ has a balanced presentation and $m_{\mathbb{H}}(G) \leq 2$, then $G$ has the D2 property.

This builds upon the work of F. E. A. Johnson Jo03a who considered the special case where there exists a 4 -periodic free resolution of $\mathbb{Z} G$ modules. In particular, for such groups $G$, he proved (i) and also obtained a version of (ii) subject to stably free $\mathbb{Z} G$ modules being free (which we resolve in Theorem 7.2 ).

This has the following consequence for the question of whether a finite Poincaré 3-complex has a cell structure with a single 3-cell:

Corollary C. Let $X$ be a finite Poincaré 3-complex with $G=\pi_{1}(X)$ finite. Then:

(i) If $X$ has a cell structure with a single 3 -cell, then $G$ has a balanced presentation.

(ii) If $G$ has a balanced presentation and $m_{\mathbb{H}}(G) \leq 2$, then $X$ has a cell structure with a single 3 -cell.

In Theorem 6.10, we show that the groups $G$ with 4-periodic cohomology for which $m_{\mathbb{H}}(G) \leq 2$ are as follows. Here we use the notation of Milnor [Mi57], and each family contains $G \times C_{n}$ for any $G$ listed and any $n \geq 1$ coprime to $|G|$.

(i) $C_{n}$ for $n \geq 1$, the cyclic groups of order $n$.

(ii) $D_{4 n+2}$ for $n \geq 1$, the dihedral groups of order $4 n+2$.

(iii) $Q_{8}, Q_{12}, Q_{16}, Q_{20}, \widetilde{T}, \widetilde{O}, \widetilde{I}$.

(iv) $D\left(2^{n}, 3\right), D\left(2^{n}, 5\right)$ for $n \geq 3$.

(v) $P_{8 \cdot 3^{n}}^{\prime}$ for $n \geq 2$.

(vi) $P_{48 n}^{\prime \prime}$ for $n \geq 3$ odd.

(vii) $Q(16 ; m, n)$ for $m>n \geq 1$ odd coprime.

By considering which of these groups have balanced presentations, we will show the following. This was previously shown by M. N. Dyer Dy75 for the groups in (i) and by Johnson Jo03a for the groups in (ii) and for many of the groups in (iii).

Theorem 8.4. Suppose $G$ is in (i)-(v) or has the form $Q(16 ; n, 1) \times C_{k}$ for some $n, k \geq 1$ odd coprime. Then $G$ has the D2 property.

Finally, we consider the case $m_{\mathbb{H}}(G) \geq 3$. Let $Q_{4 n}$ denote the quaternion group of order $4 n$, which has 4-periodic cohomology and $m_{\mathbb{H}}\left(Q_{4 n}\right)=\lfloor n / 2\rfloor$. By combining recent results of W. H. Mannan and T. Popiel [MP21] with Theorem 2.1, we show:

Theorem 8.11, $Q_{28}$ has the D2 property and $m_{\mathbb{H}}\left(Q_{28}\right)=3$.

This group was proposed as a counterexample in [BW05] (see also [MR18, p23]). We also point out that the example of Mannan-Popiel gives a counterexample to one part of the conjecture of J. M. Cohen mentioned previously Wa79a, p381]. The 
possibility remains that some group with 4-periodic cohomology does not have a balanced presentation and so would be a counterexample to the D2 problem.

We now proceed to outline the series of results which will lead to the proof of Theorem A. From now on, we will take $G$ to be a finitely presented group and we will assume all $\mathbb{Z} G$ modules are finitely generated left modules.

1.1. Reduction to algebra. Let $\mathrm{D} 2(G)$ denote the set of polarised homotopy types of finite D2 complexes $X$ with $\pi_{1}(X)=G$. This is a graded graph with grading given by the Euler characteristic $\chi(X)$ and edges between between each $(X, \rho)$ and $\left(X \vee S^{2}, \rho^{+}\right)$where $\rho^{+}$is induced by the collapse map $X \vee S^{2} \rightarrow X$.

Let $\operatorname{Alg}(G, 2)$ denote the set of chain homotopy classes of algebraic 2-complexes over $\mathbb{Z} G$, which are chain complexes $\left(F_{*}, \partial_{*}\right)$ of the form

$$
F_{2} \stackrel{\partial_{2}}{\longrightarrow} F_{1} \stackrel{\partial_{1}}{\longrightarrow} F_{0}
$$

where the $F_{i}$ are free and $H_{0}\left(F_{*}\right) \cong \mathbb{Z}$ where $\mathbb{Z}$ has trivial $G$-action. This is a graded graph with grading $\chi(E)=\operatorname{rank}\left(F_{2}\right)-\operatorname{rank}\left(F_{1}\right)+\operatorname{rank}\left(F_{0}\right)$ and edges between each $E=\left(F_{*}, \partial_{*}\right)$ and the stabilised complex $E \oplus \mathbb{Z} G=\left(F_{2} \oplus \mathbb{Z} G \stackrel{\left(\partial_{2}, 0\right)}{\longrightarrow} F_{1} \stackrel{\partial_{1}}{\longrightarrow} F_{0}\right)$.

In Section 2, we will establish the following:

Theorem 2.1. Let $G$ be a finitely presented group. Then there exists an isomorphism of graded trees

$$
\widetilde{C}_{*}: \mathrm{D} 2(G) \rightarrow \operatorname{Alg}(G, 2)
$$

which is the same as the cellular chain map $X \mapsto C_{*}(\widetilde{X})$ when $X$ is a 2-complex.

This is presumably well known (see, for example, Wa66, Theorem 4]), though we could not locate an equivalent statement in the literature. The proof we give is an adaption of the proof of the Realisation Theorem of Johnson Jo03b.

Recall that, if $P$ is a projective $\mathbb{Z} G$ module, then $P \otimes \mathbb{Q} \cong \mathbb{Q} G^{r}$ is a free $\mathbb{Q} G$ module $[\mathrm{Sw} 60 \mathrm{a}$, and we define the $\operatorname{rank}$ of $P$ to be $\operatorname{rank}(P)=r$. Let $C(\mathbb{Z} G)$ denote the projective class group and observe that a class $[P] \in C(\mathbb{Z} G)$ can also be viewed as the set of (non-zero) projective $\mathbb{Z} G$ modules $P_{0}$ for which $P \oplus \mathbb{Z} G^{i} \cong P_{0} \oplus \mathbb{Z} G^{j}$ for some $i, j \geq 0$. This has the structure of a graded graph with grading given by $\operatorname{rank}\left(P_{0}\right)$ and edges between each $P_{0}$ and $P_{0} \oplus \mathbb{Z} G$.

For a group $G$ with $n$-periodic cohomology, let $\sigma_{n}(G) \in C(\mathbb{Z} G) / T_{G}$ denote the Swan finiteness obstruction where $T_{G} \leq C(\mathbb{Z} G)$ denotes the Swan subgroup (see Section 3). By adapting an approach of Johnson [Jo03a, Theorem 62.1], we show:

Theorem 4.1. Let $G$ have 4-periodic cohomology. Then there is an isomorphism of graded trees

$$
\Psi: \operatorname{Alg}(G, 2) \rightarrow\left[P_{G}\right]
$$

for any projective $\mathbb{Z} G$ module $P_{G}$ for which $\sigma_{4}(G)=\left[P_{G}\right] \in C(\mathbb{Z} G) / T_{G}$.

An explicit description for the map $\Psi$ can be found in Proposition 4.12

1.2. Cancellation for projective modules. If $P$ is a projective $\mathbb{Z} G$ module, then we say that $[P]$ has cancellation if $P_{1} \oplus \mathbb{Z} G \cong P_{2} \oplus \mathbb{Z} G$ implies $P_{1} \cong P_{2}$ for all $P_{1}, P_{2} \in[P]$. It was shown by H. Jacobinski [Ja68, Theorem 4.1] that $[P]$ has cancellation provided $G$ is finite and satisfies the Eichler condition, i.e. $m_{\mathbb{H}}(G)=0$. The case $m_{\mathbb{H}}(G) \neq 0$ was studied extensively by Swan [Sw62, Sw83]. However, in spite of the wide applicability of these results, the only groups with 4-periodic cohomology to which they apply are the groups in (i) and (ii). 
The main contribution of this article is the following general cancellation theorem for projective $\mathbb{Z} G$ modules when $G$ is a finite group. Let $\operatorname{LF}_{1}(\mathbb{Z} G)$ denote the set of projective $\mathbb{Z} G$ modules of rank one and recall from algebraic K-theory that $K_{1}(\mathbb{Z} G)=\mathrm{GL}(\mathbb{Z} G)^{a b}$ where $\mathrm{GL}(\mathbb{Z} G)=\bigcup_{n} \mathrm{GL}_{n}(\mathbb{Z} G)$.

Theorem 5.1. Let $G$ be a finite group with quotient $H=G / N$, let $\bar{P} \in \operatorname{LF}_{1}(\mathbb{Z} G)$ and let $P=\bar{P} \otimes_{\mathbb{Z N}} \mathbb{Z} \in \mathrm{LF}_{1}(\mathbb{Z} H)$. Suppose $m_{\mathbb{H}}(G)=m_{\mathbb{H}}(H)$ and that the map

$$
\operatorname{Aut}(P) \rightarrow K_{1}(\mathbb{Z} H)
$$

is surjective. Then $[\bar{P}]$ has cancellation if and only if $[P]$ has cancellation.

This generalises a result of the author [Ni21, Theorem A] which dealt with the case of stably free $\mathbb{Z} G$ modules, i.e. where $\bar{P}=\mathbb{Z} G$ and $P=\mathbb{Z} H$.

Our first application generalises the main result in $\mathrm{Sw83}$. We say that $\mathbb{Z} G$ has stably free cancellation (SFC) if every stably free $\mathbb{Z} G$ module is free or, equivalently, if the class $[\mathbb{Z} G]$ has cancellation.

Theorem 7.1, Let $G$ have periodic cohomology. Then $\mathbb{Z} G$ has SFC if and only if $m_{\mathbb{H}}(G) \leq 2$.

This completely determines the groups $G$ with periodic cohomology for which $\mathbb{Z} G$ has SFC and also corrects a mistake in [Jo03a, p249] where it was suggested that the groups in (vii) did not have SFC. Our second application is the following:

Theorem 7.2, Let $G$ have 4-periodic cohomology and let $P_{G}$ be a projective $\mathbb{Z} G$ module for which $\sigma_{4}(G)=\left[P_{G}\right] \in C(\mathbb{Z} G) / T_{G}$. Then $\left[P_{G}\right]$ has cancellation if and only if $m_{\mathbb{H}}(G) \leq 2$.

By Theorems 2.1 and 4.1, D2 $(G)$ is isomorphic to $\left[P_{G}\right]$ as a graded tree. Hence Theorem 7.2 implies that D2 $(G)$ has cancellation if and only if $m_{\mathbb{H}}(G) \leq 2$. This completes the proof of Theorem $\mathrm{A}$. For a more detailed argument, see Section 7 .

\section{Polarised homotopy types and Algebraic 2-complexes}

The aim of this section will be to prove the following. Our proof will be a mild generalisation of the arguments of Johnson in [Jo03b].

Theorem 2.1. Let $G$ be a finitely presented group. Then there exists an isomorphism of graded trees

$$
\widetilde{C}_{*}: \mathrm{D} 2(G) \rightarrow \operatorname{Alg}(G, 2)
$$

which is the same as the cellular chain map $X \mapsto C_{*}(\tilde{X})$ when $X$ is a 2-complex.

We begin by noting that every finite D2 complex is a finite D3 complex and so is homotopy equivalent to a finite 3-complex by Wa65, Theorem E]. We therefore lose no generality in assuming throughout that every finite D2 complex is a finite 3 -complex. Let $(X, \rho) \in \mathrm{D} 2(G)$ and consider the cellular chain complex

$$
C_{*}(X)=\left(C_{3}(\tilde{X}) \stackrel{\partial_{3}}{\longrightarrow} C_{2}(\tilde{X}) \stackrel{\partial_{2}}{\longrightarrow} C_{1}(\tilde{X}) \stackrel{\partial_{1}}{\longrightarrow} C_{0}(\tilde{X})\right)
$$

where the $C_{i}(\tilde{X})$ are free $\mathbb{Z}\left[\pi_{1}(X)\right]$ modules under the monodromy action. We can use $\rho$ to identify this with a chain complex of $\mathbb{Z} G$ modules which we denote $C_{*}(X, \rho)$. We will now show the following which is also our definition for $\widetilde{C}_{*}$ :

Proposition 2.2. Let $(X, \rho) \in \mathrm{D} 2(G)$. Then $C_{*}(X, \rho)$ is chain homotopy equivalent to an algebraic 2-complex $E$ over $\mathbb{Z} G$. In particular, we define $\widetilde{C}_{*}(X, \rho)=E$. 
In order to prove this, we will need the following two lemmas. Note that, since $\operatorname{Im}\left(\partial_{3}\right) \subseteq \operatorname{ker}\left(\partial_{2}\right)$, there is a well-defined map $\widetilde{\partial}_{2}: C_{2}(\widetilde{X}) / \operatorname{Im}\left(\partial_{3}\right) \rightarrow C_{1}(\widetilde{X})$.

Lemma 2.3 ([Jo3b Proposition 6.6]). Let $(X, \rho) \in \mathrm{D} 2(G)$. Then there is a chain homotopy equivalence $\varphi: C_{*}(X, \rho) \rightarrow C_{*}^{\prime}(X, \rho)$ where

$$
C_{*}^{\prime}(X, \rho)=\left(C_{2}(\tilde{X}) / \operatorname{Im}\left(\partial_{3}\right) \stackrel{\widetilde{\partial}_{2}}{\longrightarrow} C_{1}(\tilde{X}) \stackrel{\partial_{1}}{\longrightarrow} C_{0}(\tilde{X})\right) .
$$

Lemma 2.4 (Jo03b, Proposition 6.5]). Let $(X, \rho) \in \mathrm{D} 2(G)$ and let $C_{*}(X, \rho)=$ $\left(C_{*}(\tilde{X}), \partial_{*}\right)_{0 \leq * \leq 3}$. Then $C_{2}(\widetilde{X}) / \operatorname{Im}\left(\partial_{3}\right)$ is a stably free $\mathbb{Z} G$ module.

Proof of Proposition 2.2. By Lemma 2.4 there exists $i, j \geq 0$ for which there is an isomorphism $f: C_{2}(\widetilde{X}) / \operatorname{Im}\left(\partial_{3}\right) \oplus \mathbb{Z} G^{i} \cong \mathbb{Z} G^{j}$. We can now define a chain homotopy equivalence

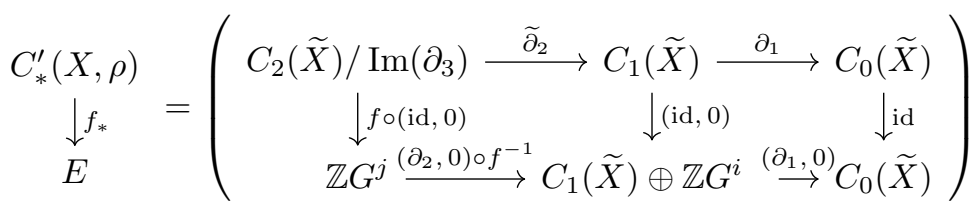

where $E$ is an algebraic 2 -complex over $\mathbb{Z} G$. Hence, by combining with Lemma 2.3, we obtain a chain homotopy equivalence $f_{*} \circ \varphi: C_{*}(X, \rho) \rightarrow E$.

We now turn to the proof of Theorem 2.1. By Proposition 2.2. $\widetilde{C}_{*}$ is a welldefined map and it is clear from the definition that $\widetilde{C}_{*}\left(X \vee S^{n}\right) \simeq \widetilde{C}_{*}(X) \oplus \mathbb{Z} G$ and so $\widetilde{C}_{*}$ gives a map of graded graphs. Note that $\operatorname{Alg}(G, 2)$ is a tree Jo03a, Section 52]. In particular, $\widetilde{C}_{*}$ is an isomorphism of graded trees if and only if it is bijective. There are many proofs in the literature that $\widetilde{C}_{*}$ is surjective (see Wa66, Theorem 4] or [Ma09, Theorem 2.1]) and so it remains to show that $\widetilde{C}_{*}$ is injective.

We will now need the following two lemmas. The first is proven in the case where $X$ and $Y$ are finite 2-complexes in Jo03b, Proposition 2.2]. The proof for finite D2 complexes is similar and so will be omitted for brevity.

Lemma 2.5. Let $\left(X, \rho_{X}\right),\left(Y, \rho_{Y}\right) \in \mathrm{D} 2(G)$ be such that $X^{(1)}=Y^{(1)}$. If $\nu$ : $C_{*}\left(X, \rho_{X}\right) \rightarrow C_{*}\left(Y, \rho_{Y}\right)$ is a chain map, then $\nu$ is chain homotopy equivalent to a chain map $\varphi$ such that $\left.\varphi\right|_{C_{i}(\widetilde{X})}=$ id for $i \leq 1$.

Let $\operatorname{PHT}(G, 2) \subseteq \mathrm{D} 2(G)$ be the subgraph corresponding to the polarised homotopy types of finite 2 -complexes.

Lemma 2.6 ([Jo03b, Lemma 2.3]). Let $\left(X, \rho_{X}\right),\left(Y, \rho_{Y}\right) \in \operatorname{PHT}(G, 2)$ be such that $X^{(1)}=Y^{(1)}$. If $\varphi: C_{*}\left(X, \rho_{X}\right) \rightarrow C_{*}\left(Y, \rho_{Y}\right)$ is a chain map such that $\left.\varphi\right|_{C_{i}(\tilde{X})}=\mathrm{id}$ for $i \leq 1$, then there exists a map $f: X \rightarrow Y$ such that $f_{*}=\varphi_{*},\left.f\right|_{X^{(1)}}=$ id and $\rho_{X}=\rho_{Y} \circ \pi_{1}(f)$.

Proof of Theorem [2.1. Let $\left(X, \rho_{X}\right),\left(Y, \rho_{Y}\right) \in \mathrm{D} 2(G)$ and note that, by the argument of [Jo03b, Proposition 2.1], we can assume that $X^{(1)}=Y^{(1)}$ by replacing each space with a polarised homotopy equivalent space. Suppose there is a chain homotopy $\widetilde{\nu}: \widetilde{C}_{*}(X) \rightarrow \widetilde{C}_{*}(Y)$. By Lemma 2.3 , this lifts to a chain homotopy $\nu: C_{*}\left(X, \rho_{X}\right) \rightarrow C_{*}\left(Y, \rho_{Y}\right)$ and, by Lemma 2.5, this is chain homotopy equivalent to a chain homotopy $\varphi: C_{*}\left(X, \rho_{X}\right) \rightarrow C_{*}\left(Y, \rho_{Y}\right)$ such that $\left.\varphi\right|_{C_{i}(\widetilde{X})}=$ id for $i \leq 1$. 
Let $i_{X}: X^{(2)} \hookrightarrow X$ denote the inclusion and note that this induces a $\mathbb{Z} G$ chain $\operatorname{map}\left(i_{X}\right)_{*}: C_{*}\left(X^{(2)}\right) \rightarrow C_{*}(X)$ where the 2-skeleton $X^{(2)}$ comes equipped with the polarisation $\rho_{X^{(2)}}=\rho_{X} \circ \pi_{1}\left(i_{X}\right)$, and similarly for $Y^{(2)}$. Since $\left(\varphi \circ i_{X}\right)_{3}=0$, the composition $\varphi_{*} \circ\left(i_{X}\right)_{*}: C_{*}\left(X^{(2)}\right) \rightarrow C_{*}(Y)$ can be viewed as a chain map

$$
\varphi_{*} \circ\left(i_{X}\right)_{*}: C_{*}\left(X^{(2)}\right) \rightarrow C_{* \leq 2}(Y) \cong C_{*}\left(Y^{(2)}\right) .
$$

Since $\left(\varphi \circ i_{X}\right)_{*}=$ id for $* \leq 1$, Lemma 2.6 implies that there exists a map $f: X^{(2)} \rightarrow$ $Y^{(2)}$ such that $f_{*}=\varphi_{*} \circ\left(i_{X}\right)_{*},\left.f\right|_{X^{(1)}}=\mathrm{id}$ and $\rho_{X^{(2)}}=\rho_{Y^{(2)}} \circ \pi_{1}(f)$. By composing with $i_{Y}$, we can assume $f: X^{(2)} \rightarrow Y$ which instead has that $\rho_{X^{(2)}}=\rho_{Y} \circ \pi_{1}(f)$.

We now claim that $f$ has an extension $F: X \rightarrow Y$ such that $F_{*}=\varphi_{*}: H_{2}(\widetilde{X}) \rightarrow$ $H_{2}(\widetilde{Y})$, which is an isomorphism since $\varphi_{*}$ is a homology equivalence. Since $X$ and $Y$ are finite D2 complexes, we have that $H_{i}(\tilde{X})=H_{i}(\widetilde{Y})=0$ for $i \neq 2$. This implies that $F$ is a homology equivalence and so is a homotopy equivalence by Whitehead's theorem. Since $F \circ i_{X}=f$ and $\rho_{X(2)}=\rho_{Y} \circ \pi_{1}(f)$, this implies that $\rho_{X}=\rho_{Y} \circ \pi_{1}(F)$ and so $F$ is the required polarised homotopy equivalence from $\left(X, \rho_{X}\right)$ to $\left(Y, \rho_{Y}\right)$.

To find the extension $F$, first let

$$
X=X^{(2)} \cup_{\alpha_{1}} e_{1}^{3} \cup_{\alpha_{2}} \cdots \cup_{\alpha_{n}} e_{n}^{3}
$$

for 3 -cells $e_{i}^{3} \cong D^{3}$ and attaching maps $\alpha_{i} \in \pi_{2}\left(X^{(2)}\right)$, where such a decomposition exists since $X$ is assumed to be a finite 3 -complex.

Using cellular chains, we have that $\partial_{3}\left(e_{i}^{3}\right)=\alpha_{i}$ where we are using the identification $\operatorname{Im}\left(\partial_{3}\right) \subseteq \operatorname{ker}\left(\partial_{2}\right) \cong \pi_{2}\left(X^{(2)}\right)$, and so $\alpha_{i} \in \operatorname{Im}\left(\partial_{3}\right)$ for all $i=1, \ldots, n$. Note that there is a commutative diagram

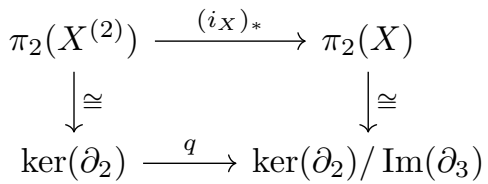

where $q$ is the quotient map. This shows that $\operatorname{Im}\left(\partial_{3}\right)=\operatorname{ker}\left(\left(i_{X}\right)_{*}\right)$. Consider the composition $f_{*}=\varphi_{*} \circ\left(i_{X}\right)_{*}: \pi_{2}\left(X^{(2)}\right) \rightarrow \pi_{2}(Y)$. Since $\varphi_{*}$ is a homology equivalence, this implies that $\operatorname{ker}\left(\left(i_{X}\right)_{*}\right)=\operatorname{ker}\left(f_{*}\right)$. By combining with the above two results, we get that $\alpha_{i} \in \operatorname{ker}\left(f_{*}\right)$ and so the maps $f \circ \alpha_{i} \in \pi_{2}(Y)$ are nullhomotopic for all $i=1, \ldots, n$.

By standard homotopy theory, this implies that there exists an extension $F$ : $X \rightarrow Y$. In particular, since $f \circ \alpha_{i}: S^{2} \rightarrow Y$ is null-homotopic, there is a map $f_{i}: e_{i}^{3} \rightarrow Y$ for which $f_{i} \circ i=f \circ \alpha_{i}$ for $i: S^{2}=\partial e_{i}^{3} \hookrightarrow e_{i}^{3}$ and so we can get a well-defined map $F: X \rightarrow Y$ by defining $\left.F\right|_{e_{i}^{3}}=f_{i}$ for each $i=1, \ldots, n$. Finally note that, by the above diagram, $\left(i_{X}\right)_{*}: \pi_{2}\left(X^{(2)}\right) \rightarrow \pi_{2}(X)$ is surjective. Since $F_{*} \circ\left(i_{X}\right)_{*}=\varphi_{*} \circ\left(i_{X}\right)_{*}$ for $* \leq 2$, this implies that $F_{*}=\varphi_{*}: \pi_{2}(X) \rightarrow \pi_{2}(Y)$ or, equivalently, that $F_{*}=\varphi_{*}: H_{2}(\tilde{X}) \rightarrow H_{2}(\tilde{Y})$.

We conclude this section by noting that Theorem 2.1 can be used to recover two well-known results on the D2 problem. Firstly, since D2 $(G)$ and $\operatorname{Alg}(G, 2)$ are isomorphic as graphs and $\operatorname{Alg}(G, 2)$ is a tree, this implies that $\mathrm{D} 2(G)$ is a tree. Since D2 $(G)$ contains a presentation complex over $G$, this leads to the following stable solution to the D2 problem which was first proved by J. M. Cohen Co77].

Corollary 2.7. If $X$ is a finite D2 complex, then there exists $n \geq 0$ for which $X \vee n S^{2}$ is homotopy equivalent to a finite 2-complex. 
Recall that an algebraic 2-complex over $\mathbb{Z} G$ is geometrically realisable if it is chain homotopy equivalent to the cellular chain complex $C_{*}(X, \rho)$ of a polarised finite 2-complex $(X, \rho)$. This begs the following question:

Realisation problem. Let $G$ be a finitely presented group. Then is every algebraic 2 -complex over $\mathbb{Z} G$ geometrically realisable?

Note that every algebraic 2-complex over $\mathbb{Z} G$ is geometrically realisable if and only if $C_{*}: \operatorname{PHT}(G, 2) \rightarrow \operatorname{Alg}(G, 2)$ is surjective. By Theorem 2.1, this is the case if and only if $\operatorname{PHT}(G, 2)=\mathrm{D} 2(G)$, i.e. if $G$ has the D2 property. This implies the following which was first proven by Johnson Jo03b subject to a mild condition on $G$ which was subsequently shown to be unnecessary by Mannan Ma09.

Corollary 2.8. A finitely presented group $G$ has the D2 property if and only if every algebraic 2 -complex over $\mathbb{Z} G$ is geometrically realisable. In particular, the $\mathrm{D} 2$ problem and Realisation problem are equivalent.

Finally, we remark that one can get an algebraic classification of the homotopy types of finite D2 complexes $X$ with $\pi_{1}(X)=G$ by quotienting $\operatorname{Alg}(G, 2)$ by the action of $\operatorname{Aut}(G)$. Finding the number of polarised homotopy types that correspond to a given homotopy type $X$ is equivalent to finding which group automorphisms $\operatorname{Aut}(G)$ are induced by self-homotopy equivalences $\mathcal{E}(X)$ (see, for example, [0165]).

\section{Projective chain complexes over Integral Group Rings}

The aim of this section will be to recall basic preliminaries on projective chain complexes and the Swan finiteness obstruction. From now on, we will assume that $G$ is a finite group.

For $\mathbb{Z} G$ modules $A$ and $B$, we define $\operatorname{hProj}_{\mathbb{Z} G}^{n}(A, B)$ to be the set of chain homotopy classes of exact sequences of $\mathbb{Z} G$ modules

$$
E=\left(0 \rightarrow B \stackrel{\iota}{\rightarrow} P_{n-1} \stackrel{\partial_{n-1}}{\longrightarrow} P_{n-2} \rightarrow \cdots \rightarrow P_{1} \stackrel{\partial_{1}}{\rightarrow} P_{0} \stackrel{\varepsilon}{\rightarrow} A \rightarrow 0\right)
$$

where the $P_{i}$ are projective. By a chain homotopy, we formally mean a chain map which restricts to a chain homotopy equivalence on the middle terms $\left(P_{*}, \partial_{*}\right)$. For brevity, we will often omit the maps $\iota$ and $\varepsilon$ in our description of $E$.

Define the Euler class to be $e(E)=\sum_{i=0}^{n-1}(-1)^{i}\left[P_{i}\right] \in C(\mathbb{Z} G)$, which only depends on the chain homotopy type of $E$. For a class $\chi \in C(\mathbb{Z} G)$, we define $\operatorname{hProj}_{\mathbb{Z} G}^{n}(A, B ; \chi)$ to be the subset of $\operatorname{hProj}_{\mathbb{Z} G}^{n}(A, B)$ consisting of those extensions with $e(E)=\chi$. Let $\Omega_{n}^{\chi}(A)$ denote the set of $\mathbb{Z} G$ modules $B$ for which $\operatorname{hProj}_{\mathbb{Z} G}^{n}(A, B ; \chi)$ is non-empty. If $n \geq 2$ then, for any $B_{0} \in \Omega_{n}^{\chi}(A)$, we have $\Omega_{n}^{\chi}(A)=\left\{B: B \oplus \mathbb{Z} G^{i} \cong B_{0} \oplus \mathbb{Z} G^{j}, i, j \geq 0\right\}$, i.e. $\Omega_{n}^{\chi}(A)$ is a stable module Sw60b. We also define

$$
\operatorname{hProj}_{\mathbb{Z} G}^{n}\left(A, \Omega_{n}^{\chi}(A) ; \chi\right)=\bigsqcup_{B \in \Omega_{n}^{\chi}(A)} \operatorname{hProj}_{\mathbb{Z} G}^{n}(A, B ; \chi)
$$

which has the structure of a graded graph with grading $\chi(E)=\sum_{i=0}^{n-1}(-1)^{i} \operatorname{rank}\left(P_{i}\right)$ and with edges between each $E$ to the stabilised complex

$$
E \oplus \mathbb{Z} G=\left(P_{n-1} \oplus \mathbb{Z} G \stackrel{\left(\partial_{n-1}, 0\right)}{\longrightarrow} P_{n-2} \rightarrow \cdots \rightarrow P_{1} \stackrel{\partial_{1}}{\longrightarrow} P_{0}\right) .
$$

We can also define $\Omega_{-n}^{\chi}(B)$ to be the set of $\mathbb{Z} G$ modules $A$ for which $\operatorname{hProj}_{\mathbb{Z} G}^{n}(A, B ; \chi)$ is non-empty and we can similarly define a graded graph $\operatorname{hProj}_{\mathbb{Z} G}^{n}\left(\Omega_{-n}^{\chi}(B), B ; \chi\right)$. 
Recall that, for a $\mathbb{Z} G$ module $A$, its dual is defined as $A^{*}=\operatorname{Hom}_{\mathbb{Z}}(A, \mathbb{Z})$ which is a left $\mathbb{Z} G$ module under the action defined by $(g \cdot \varphi)(x)=\varphi\left(g^{-1} \cdot x\right)$ for $\varphi \in A^{*}$ and $g \in G$. For $\chi \in C(\mathbb{Z} G)$, we will write $\Omega_{n}^{\chi}(A)^{*}=\left\{B^{*}: B \in \Omega_{n}^{\chi}(A)\right\}$. Recall also that a $\mathbb{Z} G$ module $A$ is a $\mathbb{Z} G$ lattice if its underlying abelian group is free, i.e. of the form $\mathbb{Z}^{n}$ for some $n \geq 0$. If $A$ is a $\mathbb{Z} G$ lattice, then $A^{*}$ is a $\mathbb{Z} G$ lattice and there is an isomorphism $\left(A^{*}\right)^{*} \cong A$ Jo03a, Section 28]. If $P$ is projective, then so is $P^{*}$ and we also have $\left(P^{*}\right)^{*} \cong P$ since projective $\mathbb{Z} G$ modules are $\mathbb{Z} G$ lattices.

If $E=\left(P_{*}, \partial_{*}\right) \in \operatorname{hProj}_{\mathbb{Z} G}^{n}(A, B)$, then define

$$
E^{*}=\left(P_{0}^{*} \stackrel{\partial_{1}^{*}}{\longrightarrow} P_{1}^{*} \rightarrow \cdots \rightarrow P_{n-2}^{*} \stackrel{\partial_{n-1}^{*}}{\longrightarrow} P_{n-1}^{*}\right) .
$$

Lemma 3.1 (Jo03a, Proposition 28.4]). Let $A$ and $B$ be $\mathbb{Z} G$ lattices. Then we have $E^{*} \in \operatorname{hProj}_{\mathbb{Z} G}^{n}\left(B^{*}, A^{*}\right)$ and $\left(E^{*}\right)^{*} \simeq E$ are chain homotopy equivalent.

By addition of elementary complexes, we can show that every projective extension $E$ with $e(E)=0$ is chain homotopy equivalent to an extension with the $P_{i}$ free provided $n \geq 2$. In particular, $\operatorname{PProj}_{\mathbb{Z} G}^{n}(A, B ; 0)$ can be taken to be the set of chain homotopy types of exact sequences $E$ with the $P_{i}$ free. We also let $\Omega_{n}(A)=\Omega_{n}^{0}(A)$.

It was shown by Swan [Sw60b, Theorem 4.1] that a group $G$ has $n$-periodic cohomology if and only if there exists an exact sequence of the form

$$
0 \rightarrow \mathbb{Z} \rightarrow P_{n-1} \rightarrow P_{n-2} \rightarrow \cdots \rightarrow P_{1} \rightarrow P_{0} \rightarrow \mathbb{Z} \rightarrow 0
$$

where the $P_{i}$ are projective, i.e. if $\operatorname{hProj}_{\mathbb{Z} G}^{n}(\mathbb{Z}, \mathbb{Z})$ is non-empty. By taking the map $P_{0} \rightarrow P_{n-1}$ which factors through $\mathbb{Z}$, this can be turned into in $n$-periodic projective resolution. We say that $G$ has free period $n$ if such a resolution exists with the $P_{i}$ free or, equivalently, if $\operatorname{hProj}_{\mathbb{Z} G}^{n}(\mathbb{Z}, \mathbb{Z} ; 0)$ is non-empty.

Recall that the Swan map $S:(\mathbb{Z} /|G|)^{\times} \rightarrow C(\mathbb{Z} G)$ sends $r \mapsto[(r, \Sigma)]$, where $\Sigma=\sum_{g \in G} g$ is the group norm and $(r, \Sigma) \subseteq \mathbb{Z} G$ has finite index coprime to $|G|$ and so is projective by [Sw60a]. We define the Swan subgroup to be $T_{G}=\operatorname{Im}(S)$. Finally, if $G$ has $n$-periodic cohomology and $E \in \operatorname{hProj}_{\mathbb{Z} G}^{n}(\mathbb{Z}, \mathbb{Z})$, then we define the Swan finiteness obstruction to be the class

$$
\sigma_{n}(G)=[e(E)] \in C(\mathbb{Z} G) / T_{G} .
$$

It was shown by Swan that $\sigma_{n}(G)$ is independent of the choice of $E$ Sw60b, Lemma 7.3]. Furthermore, we have:

Theorem 3.2 ([Sw60b]). Let $G$ have n-periodic cohomology. Then the following are equivalent:

(i) $G$ has free period $n$.

(ii) $\sigma_{n}(G)=0 \in C(\mathbb{Z} G) / T_{G}$.

(iii) There is a finite $C W$-complex $X$ such that $X \simeq S^{n-1}$ and $G$ acts freely on $X$.

It took over 20 years until the first example of a group with $\sigma_{n}(G) \neq 0$ was found by R. J. Milgram [Mi85. It was later shown by J. F. Davis Da82 that the group $Q(16 ; 3,1)$ with 4-periodic cohomology has free period 8 , which is the example of minimal order. Conversely, we also have:

Proposition 3.3 ([Sw83, Lemma 7.4]). Let $G$ have $n$-periodic cohomology and let $P_{G}$ be a projective $\mathbb{Z} G$ module for which $\sigma_{n}(G)=\left[P_{G}\right] \in C(\mathbb{Z} G) / T_{G}$. Then there exists $E \in \operatorname{hProj}_{\mathbb{Z} G}^{n}(\mathbb{Z}, \mathbb{Z})$ for which $e(E)=\left[P_{G}\right] \in C(\mathbb{Z} G)$.

The formulation (iii) has the following consequence for finite Poincaré 3-complexes $X$ since, if $\pi_{1}(X)$ is finite, then $\widetilde{X} \simeq S^{3}$. 
Corollary 3.4. A finite group $G$ is the fundamental group of a finite Poincaré 3 -complex if and only if $G$ has free period 4 .

\section{Classification of algebraic 2-COmplexes}

This section will largely be dedicated to the proof of the following theorem from the introduction.

Theorem 4.1. Let $G$ have 4-periodic cohomology. Then there is an isomorphism of graded trees

$$
\Psi: \operatorname{Alg}(G, 2) \rightarrow\left[P_{G}\right]
$$

for any projective $\mathbb{Z} G$ module $P_{G}$ for which $\sigma_{4}(G)=\left[P_{G}\right] \in C(\mathbb{Z} G) / T_{G}$.

A similar statement appears in [Jo03a, Theorem 57.4] though, due to a gap in the proof of Theorem 56.9, the argument given only applies in the case of minimal algebraic 2-complexes. Furthermore, the argument that $\{E \in \operatorname{Alg}(G, 2): \chi(E)=r\}$ and $\left\{P \in\left[P_{G}\right]: \operatorname{rank}(P)=r\right\}$ are in one-to-one correspondence for all $r \geq 1$ assumes that the former set is non-empty for each $r$, and this was only subsequently shown when $G$ has free period 4 [Jo03a, p234].

Let $\mu_{2}(G)$ be the minimum value of $\chi(E)$ over all $E \in \operatorname{Alg}(G, 2)$. Since $G$ is finite, we have $\mu_{2}(G) \geq 1$ [Sw65, Corollary 1.3]. We begin by showing the following:

Proposition 4.2. If $G$ has 4-periodic cohomology, then $\mu_{2}(G)=1$.

Recall that the augmentation ideal is the module $I=\operatorname{ker}(\varepsilon: \mathbb{Z} G \rightarrow \mathbb{Z})$ where $\varepsilon: \mathbb{Z} G \rightarrow \mathbb{Z}$ sends $g \mapsto 1$ for all $g \in G$. We will need the following three lemmas.

Lemma 4.3. Let $G$ be a finite group and let $\alpha: \mathbb{Z} \rightarrow \mathbb{Z} G^{n}$ be injective. If $\operatorname{coker}(\alpha)$ is a $\mathbb{Z} G$ lattice, then $\operatorname{coker}(\alpha) \cong I^{*} \oplus \mathbb{Z} G^{n-1}$ where $I$ is the augmentation ideal.

Proof. The case $n=1$ follows from the fact that $\alpha=r \Sigma$ for $r \neq 0$ since $\mathbb{Z} G / \Sigma \cong I^{*}$, and the case $n \geq 2$ follows from [Jo03a, Proposition 29.2].

Lemma 4.4. Let $P$ be a projective $\mathbb{Z} G$-module, let $r=\operatorname{rank}(P)$ and let $I$ be the augmentation ideal. Then there exists a $\mathbb{Z} G$ lattice $J$ for which

$$
I \oplus P=J \oplus \mathbb{Z} G^{r} .
$$

Proof. Since $G$ is a finite, $[\mathrm{Sw60a}$, Theorem A] implies that $P$ is of the form $P=$ $P_{0} \oplus \mathbb{Z} G^{r-1}$ for some rank one projective $\mathbb{Z} G$ module $P_{0}$ and so it suffices to prove the case $r=1$. Let $\varphi: P \rightarrow \mathbb{Z}$ be the surjection obtained by taking the composition

$$
P \rightarrow P \otimes \mathbb{Q} \cong \mathbb{Q} G \rightarrow \mathbb{Q}
$$

whose image is a non-trivial finitely-generated subgroup of $\mathbb{Q}$, and so isomorphic to $\mathbb{Z}$. If $J=\operatorname{ker}(\varphi)$ then, by applying Schanuel's lemma to the exact sequences

$$
0 \rightarrow I \rightarrow \mathbb{Z} G \rightarrow \mathbb{Z} \rightarrow 0, \quad 0 \rightarrow J \rightarrow P \rightarrow \mathbb{Z} \rightarrow 0,
$$

we get that $I \oplus P \cong J \oplus \mathbb{Z} G$.

Lemma 4.5 (Wa79b, p514]). Let $J$ be a $\mathbb{Z} G$ lattice. Then $\operatorname{Ext}_{\mathbb{Z} G}^{1}(J, \mathbb{Z} G)=0$.

Proof of Proposition 4.2. Since $G$ has 4-periodic cohomology, the discussion in Section 3 implies that there exists an exact sequence of $\mathbb{Z} G$-modules

$$
0 \rightarrow \mathbb{Z} \stackrel{\alpha}{\rightarrow} F_{3} \rightarrow P_{2} \rightarrow F_{1} \rightarrow F_{0} \rightarrow \mathbb{Z} \rightarrow 0
$$


where $P_{2}$ is projective and, by addition of elementary complexes, we can assume the $F_{i}$ are free. By Lemma 4.3, $\operatorname{coker}(\alpha) \cong I^{*} \oplus \mathbb{Z} G^{r}$ where $r=\operatorname{rank}\left(F_{3}\right)-1$. This gives an exact sequence:

$$
0 \rightarrow I^{*} \oplus \mathbb{Z} G^{r} \rightarrow P_{2} \rightarrow F_{1} \rightarrow F_{0} \rightarrow \mathbb{Z} \rightarrow 0 .
$$

Now let $\bar{P}_{2}$ be a projective for which $F_{2}=P_{2} \oplus \bar{P}_{2}$ is free. By forming the direct sum with length two exact sequence, we get

$$
0 \rightarrow I^{*} \oplus P \rightarrow F_{2} \rightarrow F_{1} \rightarrow F_{0} \rightarrow \mathbb{Z} \rightarrow 0
$$

for $P=\mathbb{Z} G^{r} \oplus \bar{P}_{2}$ projective. By dualising the result in Lemma 4.4, we can write $I^{*} \oplus P=J \oplus \mathbb{Z} G^{s}$ where $s=\operatorname{rank}(P)$.

Let $i$ denote the injection $i: J \oplus \mathbb{Z} G^{s} \cong I^{*} \oplus P \rightarrow F_{2}$, and consider the exact sequences:

$$
0 \rightarrow J \rightarrow F_{2} / i\left(\mathbb{Z} G^{s}\right) \rightarrow F_{1} \rightarrow F_{0} \rightarrow \mathbb{Z} \rightarrow 0, \quad 0 \rightarrow \mathbb{Z} G^{s} \rightarrow F_{2} \rightarrow F_{2} / i\left(\mathbb{Z} G^{s}\right) \rightarrow 0 .
$$

The first exact sequence implies that $F_{2} / i\left(\mathbb{Z} G^{s}\right)$ is a $\mathbb{Z} G$ lattice. By Lemma 4.5 . this implies that $\operatorname{Ext}_{\mathbb{Z} G}^{1}\left(F_{2} / i\left(\mathbb{Z} G^{s}\right), \mathbb{Z} G^{s}\right)=0$ and so $F_{2} \cong F_{2} / i\left(\mathbb{Z} G^{s}\right) \oplus \mathbb{Z} G^{s}$ by the second exact sequence. Hence we get an exact sequence

$$
0 \rightarrow J \rightarrow F_{2} \rightarrow F_{1} \oplus \mathbb{Z} G^{s} \rightarrow F_{0} \rightarrow \mathbb{Z} \rightarrow 0
$$

which defines an algebraic 2-complex $E$. Since $J \cong I^{*} \cong \mathbb{Z} G^{|G|-1}$ as abelian groups, we have that $\chi(E)=1$ which completes the proof.

Recall that a graded tree is a fork if it has a single vertex at each non-minimal level (i.e. grade). The following was shown by W. H. Browning. These results were never published, though an alternate proof can be found in [Ham19, Corollary 2.6].

Theorem 4.6 ([Br78, Theorem 5.4]). Let $G$ be a finite group. Then $\operatorname{Alg}(G, 2)$ is a fork.

On the other hand, if $G$ is a finite group, then [Sw60a, Theorem A] implies that every projective $\mathbb{Z} G$ module $P$ is of the form $P=P_{0} \oplus \mathbb{Z} G^{r}$ for some rank one projective $\mathbb{Z} G$ module $P_{0}$. This implies that $[P]$ is also a fork.

Hence, in order to prove Theorem 4.1 it suffices to prove that there is a bijection $\Psi$ between $\operatorname{Alg}(G, 2)$ and $\left[P_{G}\right]$ at the minimal levels, i.e. that there is a one-to-one correspondence between $\{E \in \operatorname{Alg}(G, 2): \chi(E)=1\}$ and $\left\{P \in\left[P_{G}\right]: \operatorname{rank}(P)=1\right\}$ (see Fig. 1). We now need the following two results.

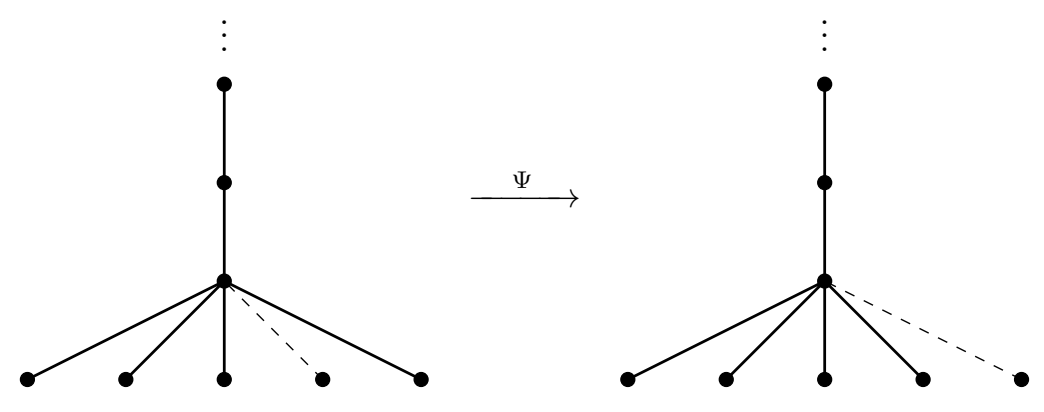

Figure 1. Tree structures for $\operatorname{Alg}(G, 2)$ and $\left[P_{G}\right]$ 
Proposition 4.7. There is an isomorphism of graded trees

$$
\operatorname{Alg}(G, 2) \cong \operatorname{hProj}_{\mathbb{Z} G}^{3}\left(\mathbb{Z}, \Omega_{3}(\mathbb{Z}) ; 0\right) .
$$

Proposition 4.8. Let $\chi=[P] \in C(\mathbb{Z} G)$. Then there is an map of graded trees

$$
\Phi: \operatorname{hProj}_{\mathbb{Z} G}^{1}\left(\Omega_{3}(\mathbb{Z}), \mathbb{Z} ; \chi\right) \rightarrow[P]
$$

given by $\left(0 \rightarrow \mathbb{Z} \rightarrow P_{0} \rightarrow J \rightarrow 0\right) \mapsto P_{0}$, which is a bijection at the minimal level.

The first is immediate from the discussion in Section 3, and the second is a consequence of the following which is a slight extension of [Jo03a, Corollary 56.5].

Lemma 4.9. For $i=1,2$, let $P_{i}$ be projective $\mathbb{Z} G$ modules of rank one and let $\mathcal{E}_{i}=\left(0 \rightarrow J \rightarrow P_{i} \rightarrow \mathbb{Z} \rightarrow 0\right)$ be exact sequences of $\mathbb{Z} G$-modules. Then there is a chain homotopy equivalence $\mathcal{E}_{1} \simeq \mathcal{E}_{2}$ if and only if $P_{1} \cong P_{2}$.

The following will be useful in comparing Propositions 4.7 and 4.8 .

Lemma 4.10. Let $G$ have 4-periodic cohomology and let $P_{G}$ be a projective $\mathbb{Z} G$ module for which $\sigma_{4}(G)=\left[P_{G}\right] \in C(\mathbb{Z} G) / T_{G}$. If $\chi=\left[P_{G}^{*}\right] \in C(\mathbb{Z} G)$, then

$$
\Omega_{3}(\mathbb{Z})=\Omega_{1}^{\chi}(\mathbb{Z})^{*} .
$$

Proof. By Proposition 3.3, there exists $E \in \operatorname{hProj}_{\mathbb{Z} G}^{4}(\mathbb{Z}, \mathbb{Z})$ with $e(E)=\left[P_{G}\right]$. By addition of elementary complexes, we can assume that

$$
E=\left(P \stackrel{\partial_{3}}{\longrightarrow} F_{2} \stackrel{\partial_{2}}{\longrightarrow} F_{1} \stackrel{\partial_{1}}{\longrightarrow} F_{0}\right)
$$

where the $F_{i}$ are free, so that $P \in\left[P_{G}\right]$.

Let $J=\operatorname{ker}\left(\partial_{2}\right)=\operatorname{Im}\left(\partial_{3}\right)$. Then $J \in \Omega_{3}(\mathbb{Z})$ and there are exact sequences

$$
\mathcal{E}=(0 \rightarrow \mathbb{Z} \stackrel{\alpha}{\rightarrow} P \stackrel{\beta}{\rightarrow} J \rightarrow 0), \quad \mathcal{E}^{*}=\left(0 \rightarrow J^{*} \stackrel{\beta^{*}}{\rightarrow} P^{*} \stackrel{\alpha^{*}}{\rightarrow} \mathbb{Z} \rightarrow 0\right),
$$

where $\mathcal{E}^{*}$ is exact by Lemma 3.1 since $\mathbb{Z}$ and $J$ are $\mathbb{Z} G$ lattices. Hence $J^{*} \in \Omega_{1}^{\chi}(\mathbb{Z})$. Since $\left(J^{*}\right)^{*} \cong J$, this implies that $J \in \Omega_{1}^{\chi}(\mathbb{Z})^{*}$. Hence $\Omega_{3}(\mathbb{Z})=\Omega_{1}^{\chi}(\mathbb{Z})^{*}$ since two stable modules are equal if they intersect non-trivially.

Recall that, if $J$ is a $\mathbb{Z} G$ module, then an automorphism $\varphi: J \rightarrow J$ induces a $\operatorname{map} \varphi_{*}: H^{n}(G ; J) \rightarrow H^{n}(G ; J)$. If $J \in \Omega_{n}(\mathbb{Z})$, then $H^{n}(G ; J) \cong \mathbb{Z} /|G|[$ Jo03a, p132]. By fixing this identification, the construction $\varphi \mapsto \varphi_{*}$ induces a map

$$
\nu^{J}: \operatorname{Aut}_{\mathbb{Z} G}(J) \rightarrow(\mathbb{Z} /|G|)^{\times} .
$$

Let $S:(\mathbb{Z} /|G|)^{\times} \rightarrow C(\mathbb{Z} G)$ denote the Swan map, as defined in Section 3 , Then:

Lemma 4.11 ([Jo03a, Theorems 54.6, 56.10]). Let $\chi \in C(\mathbb{Z} G)$ and $J \in \Omega_{n}^{\chi}(\mathbb{Z})$. Then $\operatorname{Im}\left(\nu^{J}\right) \subseteq \operatorname{ker}(S)$ and there is a bijection

$$
\operatorname{hProj}_{\mathbb{Z} G}^{n}(\mathbb{Z}, J ; \chi) \cong \operatorname{ker}(S) / \operatorname{Im}\left(\nu^{J}\right) .
$$

In particular, $\operatorname{hProj}_{\mathbb{Z} G}^{n}(\mathbb{Z}, J ; \chi)$ only depends on $J$ and not on $n$ or $\chi$.

Proof of Theorem 4.1. First note, since the map $P \mapsto P^{*}$ is an involution on the class of projective $\mathbb{Z} G$ modules, it must induce an isomorphism of graded trees $\left[P_{G}\right] \cong\left[P_{G}^{*}\right]$. Hence, by Propositions 4.7 and 4.8 it suffices to prove that the graded trees

$$
\operatorname{hProj}_{\mathbb{Z} G}^{3}\left(\mathbb{Z}, \Omega_{3}(\mathbb{Z}) ; 0\right), \quad \operatorname{hProj}_{\mathbb{Z} G}^{1}\left(\mathbb{Z}, \Omega_{1}^{\chi}(\mathbb{Z}) ; \chi\right)
$$

contain the same number of extensions at the minimal level, where $\chi=\left[P_{G}^{*}\right]$. 
To see this, let $J \in \Omega_{3}(\mathbb{Z})$ be minimal and note that $\operatorname{Aut}_{\mathbb{Z} G}(J) \cong \operatorname{Aut}_{\mathbb{Z} G}\left(J^{*}\right)$ and so there is a bijection $\operatorname{Im}\left(\nu^{J}\right) \cong \operatorname{Im}\left(\nu^{J^{*}}\right)$. In particular, we have bijections

$$
\operatorname{hProj}_{\mathbb{Z} G}^{3}(\mathbb{Z}, J ; 0) \cong \operatorname{ker}(S) / \operatorname{Im}\left(\nu^{J}\right) \simeq \operatorname{ker}(S) / \operatorname{Im}\left(\nu^{J^{*}}\right) \cong \operatorname{hProj}_{\mathbb{Z} G}^{1}\left(\mathbb{Z}, J^{*} ; \chi\right) .
$$

By Lemma 4.10, the map $J \mapsto J^{*}$ induces a bijection $\Omega_{3}(\mathbb{Z}) \cong \Omega_{1}^{\chi}(\mathbb{Z})$. We can now extend the bijection $\operatorname{hProj}_{\mathbb{Z} G}^{3}(\mathbb{Z}, J ; 0) \cong \operatorname{hProj}_{\mathbb{Z} G}^{1}\left(\mathbb{Z}, J^{*} ; \chi\right)$ over all $J \in \Omega_{3}(\mathbb{Z})$ at the minimal level, and this completes the proof.

We conclude this section by remarking that, whilst it will not be needed in the proof of Theorem A, we can obtain the following explicit form for the map $\Psi$.

Proposition 4.12. Let $G$ have 4-periodic cohomology, let $\bar{E} \in \operatorname{hProj}_{\mathbb{Z} G}^{4}(\mathbb{Z}, \mathbb{Z})$ and let $P_{G}$ be a projective $\mathbb{Z} G$ module for which $e(\bar{E})=\left[P_{G}\right]$. Then there is an isomorphism of graded trees

$$
\Psi: \operatorname{Alg}(G, 2) \rightarrow\left[P_{G}\right]
$$

sending $E=\left(F_{*}, \partial_{*}\right) \mapsto P$, where $P$ is the unique projective $\mathbb{Z} G$ module for which

$$
\left(0 \rightarrow \mathbb{Z} \stackrel{\alpha}{\rightarrow} P \stackrel{\beta}{\rightarrow} F_{2} \stackrel{\partial_{2}}{\rightarrow} F_{1} \stackrel{\partial_{1}}{\rightarrow} F_{0} \stackrel{\partial_{0}}{\rightarrow} \mathbb{Z} \rightarrow 0\right) \simeq \bar{E}
$$

is a chain homotopy equivalence for some $\alpha$ and $\beta$.

Proof. It follows from Wa79b, Lemma 1.1] that, for each $E=\left(F_{*}, \partial_{*}\right)$ and $J=$ $\operatorname{ker}\left(\partial_{2}\right) \subseteq F_{2}$, there is a bijection

$$
\begin{gathered}
\varphi_{E}: \operatorname{hProj}_{\mathbb{Z} G}^{1}(J, \mathbb{Z}) \rightarrow \operatorname{hProj}_{\mathbb{Z} G}^{4}(\mathbb{Z}, \mathbb{Z}) \\
(0 \rightarrow \mathbb{Z} \stackrel{\alpha}{\rightarrow} P \stackrel{\beta}{\rightarrow} J \rightarrow 0) \mapsto\left(0 \rightarrow \mathbb{Z} \stackrel{\alpha}{\rightarrow} P \stackrel{\beta}{\rightarrow} F_{2} \stackrel{\partial_{2}}{\rightarrow} F_{1} \stackrel{\partial_{1}}{\rightarrow} F_{0} \stackrel{\partial_{0}}{\longrightarrow} \mathbb{Z} \rightarrow 0\right) .
\end{gathered}
$$

Hence, for $\chi=\left[P_{G}\right]$, we have well-defined maps of graded trees

$$
\Psi: \operatorname{Alg}(G, 2) \rightarrow \operatorname{hProj}_{\mathbb{Z} G}^{1}\left(\Omega_{3}(\mathbb{Z}), \mathbb{Z} ; \chi\right) \rightarrow\left[P_{G}\right]
$$

where the first map is given by sending $E \mapsto \varphi_{E}^{-1}(\bar{E})$, and the second map sends $(0 \rightarrow \mathbb{Z} \stackrel{\alpha}{\rightarrow} P \stackrel{\beta}{\rightarrow} J \rightarrow 0) \mapsto P$ which is contained in $\left[P_{G}\right]$ since $e(\bar{E})=\left[P_{G}\right]$ only depends on the chain homotopy type of $\bar{E}$. It follows by tracing through the proof of Theorem 4.1 that this is a bijection and so is an isomorphism of graded trees.

In a subsequent article Ni20, we will explore the correspondence given in Proposition 4.12 in more detail and in a more general form.

\section{Cancellation for Projective modules over integral Group Rings}

Recall from algebraic K-theory that $K_{0}(\mathbb{Z} G)$ is defined as the Grothendieck group of the monoid of isomorphism classes of projective $\mathbb{Z} G$-modules $P(\mathbb{Z} G)$, i.e. the abelian group generated by $[P]$ for $P \in P(\mathbb{Z} G)$ with relations $\left[P_{1} \oplus P_{2}\right]=\left[P_{1}\right] \oplus\left[P_{2}\right]$. Recall also that $K_{1}(\mathbb{Z} G)=\mathrm{GL}(\mathbb{Z} G)^{\text {ab }}$ where $\mathrm{GL}(\mathbb{Z} G)=\bigcup_{n} \mathrm{GL}_{n}(\mathbb{Z} G)$.

For a projective $\mathbb{Z} G$ module $P$, let $\operatorname{Aut}(P)$ denote the group of $\mathbb{Z} G$ module automorphisms of $P$ and define $\operatorname{Aut}(P) \rightarrow K_{1}(\mathbb{Z} G)$ by choosing a projective $Q$ such that $P \oplus Q \cong \mathbb{Z} G^{r}$ is free and then forming the composition

$$
\operatorname{Aut}(P) \subseteq \operatorname{Aut}(P \oplus Q) \cong \mathrm{GL}_{r}(\mathbb{Z} H) \subseteq \mathrm{GL}(\mathbb{Z} H) \rightarrow K_{1}(\mathbb{Z} H) .
$$

This is well-defined by Mi71, Lemma 3.2].

The aim of this section will be to prove the following. 
Theorem 5.1. Let $G$ be a finite group with quotient $H=G / N$, let $\bar{P} \in \operatorname{LF}_{1}(\mathbb{Z} G)$ and let $P=\bar{P} \otimes_{\mathbb{Z N N}} \mathbb{Z} \in \mathrm{LF}_{1}(\mathbb{Z} H)$. Suppose $m_{\mathbb{H}}(G)=m_{\mathbb{H}}(H)$ and that the map

$$
\operatorname{Aut}(P) \rightarrow K_{1}(\mathbb{Z} H)
$$

is surjective. Then $[\bar{P}]$ has cancellation if and only if $[P]$ has cancellation.

Our proof will follow a similar outline to [Ni21, Theorem A], which established this result in the special case where $\bar{P}=\mathbb{Z} G$ and $P=\mathbb{Z} H$.

5.1. Preliminaries on locally free modules. From now on, we will let $A$ be a finite-dimensional semisimple $\mathbb{Q}$-algebra and we will let $\Lambda$ be a $\mathbb{Z}$-order in $A$, i.e. a finitely-generated subring of $A$ such that $\mathbb{Q} \cdot \Lambda=A$. For example, we can take $\Lambda=\mathbb{Z} G$ and $A=\mathbb{Q} G$. For a prime $p$, let $\Lambda_{p}=\Lambda \otimes \mathbb{Z}_{p}$ where $\mathbb{Z}_{p}$ is the $p$-adic integers. We say that a $\Lambda$ module $M$ is locally free of rank $n$ if $M \otimes \mathbb{Z}_{p}$ is a free $\Lambda_{p}$ module of rank $n$ for all primes $p$.

Let $C(\Lambda)$ denote the locally free class group, i.e. the equivalence classes of locally free $\Lambda$ modules where $P_{1} \sim P_{2}$ if $P_{1} \oplus \Lambda^{i} \cong P_{2} \oplus \Lambda^{j}$ for some $i, j \geq 0$, and we let $\mathrm{LF}_{1}(\Lambda)$ denote the set of isomorphism classes of locally free $\Lambda$ modules of rank one. We say that $\Lambda$ has locally free cancellation if $[P]$ has cancellation for all locally free $\Lambda$ modules $P$. By [Sw80, Lemma 2.1], every locally free $\Lambda$ module is projective. The converse holds in the case where $\Lambda=\mathbb{Z} G$ by [Sw70, Theorems 2.21, 4.2] and so $C(\mathbb{Z} G)$ and $\mathrm{LF}_{1}(\mathbb{Z} G)$ coincide with our previous definitions.

Let $m_{\mathbb{H}}(\Lambda)$ denote the number of copies of $\mathbb{H}$ in the Wedderburn decomposition of $\Lambda_{\mathbb{R}}=\Lambda \otimes \mathbb{R}$, and we say that $\Lambda$ satisfies the Eichler condition if $m_{\mathbb{H}}(\Lambda)=0$. The following two results will be essential to the proof of Theorem 5.1

Theorem 5.2 ([Ja68, Theorem 4.1]). Suppose $\Lambda$ satisfies the Eichler condition. Then $\Lambda$ has locally free cancellation.

Theorem 5.3 ( $\underline{\text { Sw80 }}$, Corollary 10.5]). Suppose $\Lambda$ satisfies the Eichler condition, $I \subseteq \Lambda$ is a two-sided ideal of finite index and $f: \Lambda \rightarrow \Lambda / I$ is the induced map. Then $f\left(\Lambda^{\times}\right) \unlhd(\Lambda / I)^{\times}$and $(\Lambda / I)^{\times} \rightarrow K_{1}(\Lambda / I)$ induces an isomorphism

$$
\frac{(\Lambda / I)^{\times}}{\Lambda^{\times}} \cong \frac{K_{1}(\Lambda / I)}{K_{1}(\Lambda)}
$$

Recall the following which is a refinement of a result of A. Fröhlich [Fr75]. This gives the forward direction of Theorem 5.1 .

Theorem 5.4 ([Sw83, Theorem A10]). Let $H=G / N$, let $\bar{P} \in \mathrm{LF}_{1}(\mathbb{Z} G)$ and let $P=\bar{P} \otimes_{\mathbb{Z} N} \mathbb{Z} \in \operatorname{LF}_{1}(\mathbb{Z} H)$. If $[\bar{P}]$ has cancellation, then $[P]$ has cancellation.

Finally, recall that every projective $\mathbb{Z} G$ module $P$ is of the form $P=P_{0} \oplus \mathbb{Z} G^{r}$ where $P_{0}$ has rank one $[\mathrm{Sw60a}$, Theorem A]. In particular, the stable class map $[\cdot]: \mathrm{LF}_{1}(\mathbb{Z} G) \rightarrow C(\mathbb{Z} G)$ is surjective and is bijective precisely when $\mathbb{Z} G$ has locally free cancellation. Furthermore, $\mathbb{Z} G$ has cancellation in the class of $[P]$ precisely when the fibre over $[P] \in C(\mathbb{Z} G)$ is trivial.

5.2. Proof of Theorem 5.1. Let $H=G / N$ where $N \leq G$ is a normal subgroup, let $\bar{P} \in \mathrm{LF}_{1}(\mathbb{Z} G)$ and let $P=\bar{P} \otimes_{\mathbb{Z} N} \mathbb{Z} \in \mathrm{LF}_{1}(\mathbb{Z} H)$. Since the converse direction was proven in Theorem 5.4 it will suffice to prove that $[P]$ has cancellation subject to the following three conditions:

(i) $[\bar{P}]$ has cancellation. 
(ii) $m_{\mathbb{H}}(G)=m_{\mathbb{H}}(H)$.

(iii) The map $\mathbb{Z} H^{\times} \rightarrow K_{1}(\mathbb{Z} H)$ is surjective.

We begin by considering the following pullback diagram for $\mathbb{Z} G$ induced by $N$ :

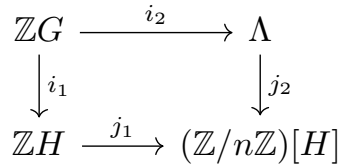

where $n=|N|, \widehat{N}=\sum_{g \in N} g$ and $\Lambda=\mathbb{Z} G / \widehat{N}$. This is the standard pullback diagram for the ring $\mathbb{Z} G$ and trivially intersecting ideals

$$
I=\operatorname{ker}(\mathbb{Z} G \rightarrow \mathbb{Z} H)=I(N) \cdot G \text { and } J=\widehat{N} \cdot \mathbb{Z} G,
$$

where $I(N)=\operatorname{ker}(\mathbb{Z} N \rightarrow \mathbb{Z})$ is the augmentation ideal [CR90, Example 42.3].

Note that $\Lambda$ is a $\mathbb{Z}$-order in $\Lambda_{\mathbb{Q}}$ which is a finite-dimensional semisimple $\mathbb{Q}$-algebra since, by tensoring the diagram with $\mathbb{Q}$, we get that $\mathbb{Q} G \cong \mathbb{Q} H \times \Lambda_{\mathbb{Q}}$. By tensoring further with $\mathbb{R}$, we get that $\mathbb{R} G \cong \mathbb{R} H \times \Lambda_{\mathbb{R}}$ and so $m_{\mathbb{H}}(G)=m_{\mathbb{H}}(H)+m_{\mathbb{H}}(\Lambda)$.

By condition (ii), $\Lambda$ satisfies the Eichler condition and so $\Lambda$ has locally free cancellation by Theorem 5.2. In particular, $\operatorname{LF}_{1}(\Lambda)=C(\Lambda)$ and, by condition (i), we also have that $\mathrm{LF}_{1}(\mathbb{Z} H) \rightarrow C(\mathbb{Z} H)$ has trivial fibre over $[P]$.

Consider the following diagram induced by the maps on projective modules.

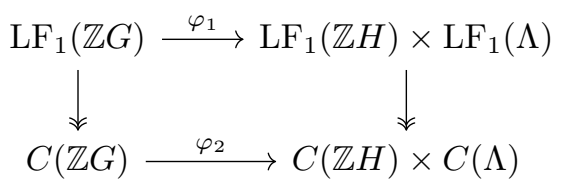

Let $Q$ be the image of $\bar{P}$ in $\operatorname{LF}_{1}(\Lambda)$. Then proving $[\bar{P}]$ has cancellation amounts to proving that the fibres of $\varphi_{1}, \varphi_{2}$ over $(P, Q)$ are in bijection. We will now compute each of these fibres in turn.

Firstly, by Theorem 8.1 of [Sw60a], we know that

$$
P \otimes(\mathbb{Z} / n \mathbb{Z})[H] \cong(\mathbb{Z} / n \mathbb{Z})[H] \cong Q \otimes(\mathbb{Z} / n \mathbb{Z})[H] .
$$

The pullback diagram for $\mathbb{Z} G$ above is a Milnor square and so, by the general construction of projectives modules using a Milnor square (see, for example, Sw80, Proposition 4.1]), the fibre $\varphi_{1}^{-1}(P, Q)$ is in correspondence with the double coset

$$
\operatorname{Aut}(P) \backslash(\mathbb{Z} / n \mathbb{Z})[H]^{\times} / \operatorname{Aut}(Q)
$$

which we take to mean $\bar{j}_{1}(\operatorname{Aut}(P)) \backslash(\mathbb{Z} / n \mathbb{Z})[H]^{\times} / \bar{j}_{2}(\operatorname{Aut}(Q))$ where $\bar{j}_{1}$ is the induced $\operatorname{map} \operatorname{Aut}(P) \rightarrow \operatorname{Aut}(P \otimes(\mathbb{Z} / n \mathbb{Z})[H]) \cong(\mathbb{Z} / n \mathbb{Z})[H]^{\times}$and similarly for $\bar{j}_{2}$.

Secondly, by [Mi71, Theorem 3.3], there is an exact sequence of the form

$$
K_{1}(\mathbb{Z} H) \times K_{1}(\Lambda) \longrightarrow K_{1}((\mathbb{Z} / n \mathbb{Z})[H]) \stackrel{\partial}{\longrightarrow} K_{0}(\mathbb{Z} G) \longrightarrow K_{0}(\mathbb{Z} H) \times K_{0}(\Lambda)
$$

where all maps other than $\partial$ are functorial, which is a part of the Mayer-Vietoris sequence for the Milnor square above.

Since projective $\mathbb{Z} G$ modules are locally free, the locally free rank induces a surjection $\mathrm{rk}: K_{0}(\mathbb{Z} G) \rightarrow \mathbb{Z}$ and, by [Sw80, p157], we have that $C(\mathbb{Z} G) \cong \operatorname{ker}(\mathrm{rk})$. 
It is now straightforward to check that $\operatorname{ker}\left(\varphi_{2}\right) \cong \operatorname{ker}\left(K_{0}(\mathbb{Z} G) \rightarrow K_{0}(\mathbb{Z} H) \times K_{0}(\Lambda)\right)$. Hence, by exactness, the fibres of $\varphi_{2}$ are in one-to-one correspondence with

$$
\operatorname{Im}\left(K_{1}((\mathbb{Z} / n \mathbb{Z})[H]) \rightarrow C(\mathbb{Z} G)\right) \cong \frac{K_{1}((\mathbb{Z} / n \mathbb{Z})[H])}{K_{1}(\mathbb{Z} H) \times K_{1}(\Lambda)}
$$

where we take the denominator to mean $\bar{j}_{1}\left(K_{1}(\mathbb{Z} H)\right)+\bar{j}_{2}\left(K_{1}(\Lambda)\right)$ where $\bar{j}_{i}=K_{1}\left(j_{i}\right)$.

Now note that $\operatorname{End}(Q)$ satisfies the Eichler condition since

$$
\operatorname{End}(Q) \otimes \mathbb{R} \cong \operatorname{End}(Q \otimes \mathbb{R}) \cong \operatorname{End}\left(\Lambda_{\mathbb{R}}\right) \cong \Lambda_{\mathbb{R}}
$$

Let $J=\operatorname{ker}(\Lambda \rightarrow(\mathbb{Z} / n \mathbb{Z})[H])$ and note that there is a map $\operatorname{End}(Q) \rightarrow \Lambda / J$ induced by localisation $[$ Sw83, p146]. This implies that $I=\operatorname{ker}(\operatorname{End}(Q) \rightarrow \Lambda / J)$ has finite index in $\operatorname{End}(Q)$ and is such that $\operatorname{End}(Q) / I \cong \Lambda / J \cong(\mathbb{Z} / n \mathbb{Z})[H]$.

Hence we can apply Theorem 5.3 to get that

$$
\frac{(\mathbb{Z} / n \mathbb{Z})[H]^{\times}}{\operatorname{Aut}(Q)} \cong \frac{K_{1}((\mathbb{Z} / n \mathbb{Z})[H])}{K_{1}(\operatorname{End}(Q))} .
$$

by using that $\operatorname{Aut}(Q)=\operatorname{End}(Q)^{\times}$. Since there is a commutative diagram

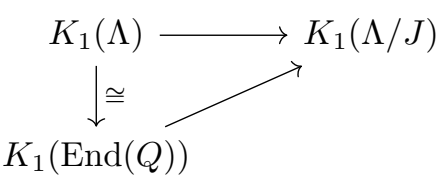

by [Sw83, Corollary A17], we get that

$$
A=\frac{(\mathbb{Z} / n \mathbb{Z})[H]^{\times}}{\operatorname{Aut}(Q)} \cong \frac{K_{1}((\mathbb{Z} / n \mathbb{Z})[H])}{K_{1}(\Lambda)}
$$

and so $\operatorname{Aut}(P) \backslash(\mathbb{Z} / n \mathbb{Z})[H]^{\times} / \operatorname{Aut}(Q)$ and $\frac{K_{1}((\mathbb{Z} / n \mathbb{Z})[H])}{K_{1}(\mathbb{Z} H) \times K_{1}(\Lambda)}$ are in correspondence if and only if the maps $\operatorname{Aut}(P) \rightarrow A$ and $K_{1}(\mathbb{Z} H) \rightarrow A$ have the same images.

Since we have a map $\operatorname{Aut}(P) \rightarrow \operatorname{Aut}_{(\mathbb{Z} / n \mathbb{Z})[H]}(P \otimes(\mathbb{Z} / n \mathbb{Z})[H]) \cong(\mathbb{Z} / n \mathbb{Z})[H]^{\times}$, we can obtain the following commutative diagram.

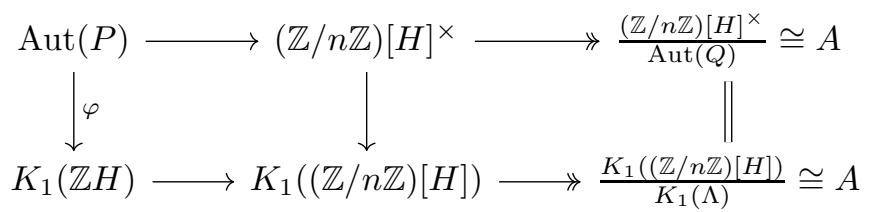

To see that the left hand square commutes, suppose $\varphi: \operatorname{Aut}(P) \rightarrow K_{1}(\mathbb{Z} H)$ is defined via $P \oplus P^{\prime} \cong \mathbb{Z} G^{r}$ for some $r \geq 1$. Since $\left(P \oplus P^{\prime}\right) \otimes(\mathbb{Z} / n \mathbb{Z})[H] \cong$ $(\mathbb{Z} / n \mathbb{Z})[H]^{r}$ and $\mathbb{Z} G^{r} \otimes(\mathbb{Z} / n \mathbb{Z})[H] \cong(\mathbb{Z} / n \mathbb{Z})[H]^{r}$, this induces an automorphism

$$
F=f \otimes(\mathbb{Z} / n \mathbb{Z})[H]:(\mathbb{Z} / n \mathbb{Z})[H]^{r} \rightarrow(\mathbb{Z} / n \mathbb{Z})[H]^{r}
$$

from which we can define a map

$$
(\mathbb{Z} / n \mathbb{Z})[H] \hookrightarrow(\mathbb{Z} / n \mathbb{Z})[H] \oplus\left(P^{\prime} \otimes(\mathbb{Z} / n \mathbb{Z})[H]\right) \cong(\mathbb{Z} / n \mathbb{Z})[H]^{r} \stackrel{F}{\rightarrow}(\mathbb{Z} / n \mathbb{Z})[H]^{r} .
$$

Since this induces a map of units $(\mathbb{Z} / n \mathbb{Z})[H]^{\times} \rightarrow \mathrm{GL}_{r}((\mathbb{Z} / n \mathbb{Z})[H])$, we can use this to define a map $(\mathbb{Z} / n \mathbb{Z})[H]^{\times} \rightarrow K_{1}((\mathbb{Z} / n \mathbb{Z})[H])$. It follows from [Mi71, Lemma 3.2] that this is the same as the map defined using the inclusion

$$
(\mathbb{Z} / n \mathbb{Z})[H]^{\times} \hookrightarrow \mathrm{GL}_{r}((\mathbb{Z} / n \mathbb{Z})[H])
$$


and so is the same as the middle vertical map in the diagram above. The left hand square then commutes by construction of the map $F$.

If $\psi_{1}: \operatorname{Aut}(P) \rightarrow A$ denotes the map along the top row and $\psi_{2}: K_{1}(\mathbb{Z} H) \rightarrow A$ denotes the map along the bottom row, then commutativity shows that $\psi_{1}=\psi_{2} \circ \varphi$. By condition (iii), $\varphi$ is surjective. Hence $\operatorname{Im} \psi_{1}=\operatorname{Im} \psi_{2}$ and so $[P]$ has cancellation. This completes the proof of Theorem 5.1 .

\section{Groups With PERIOdiC COHOMOLOGY}

The aim of this section will be to find restrictions on the quotients of groups with periodic cohomology which will allow us to apply Theorem 5.1 in Section 7 We will also classify the groups $G$ with 4-periodic cohomology for which $m_{\mathbb{H}}(G) \leq 2$, from which we will obtain the list (i)-(vii) stated in the introduction.

Recall that a group $G$ has $n$-periodic cohomology if the Tate cohomology groups $\widehat{H}^{*}(G ; \mathbb{Z})$ are periodic of period $n$.

Theorem 6.1 ([EE56, Theorem 11.6]). Let $G$ be a finite group. Then the following are equivalent:

(i) $G$ has periodic cohomology.

(ii) $G$ has no subgroup of the form $C_{p}^{2}$ for $p$ prime.

(iii) The Sylow subgroups of $G$ are cyclic or (generalised) quaternionic $Q_{2^{n}}$.

Recall that a binary polyhedral group is a finite non-cyclic subgroup of $S^{3}$ and consists of the quaternion groups $Q_{4 n}$ for $n \geq 2$ and the binary tetrahedral, octahedral and icosahedral groups $\widetilde{T}, \widetilde{O}, \widetilde{I}$. The following is well known:

Proposition 6.2 ([Ni21, Proposition 1.3]). A finite group $G$ satisfies the Eichler condition if and only if $G$ has no quotient which is a binary polyhedral group.

We now aim to prove the following, which is the main result of this section.

Theorem 6.3. Suppose $G$ has periodic cohomology and does not satisfy the Eichler condition. Then $G$ either has a quotient of the form $Q_{4 n}$ for $n \geq 6$ or a binary polyhedral quotient $H$ for which $m_{\mathbb{H}}(G)=m_{\mathbb{H}}(H)$.

In order to prove this, we will need:

Proposition 6.4 ([Ni21, Proposition 3.3]). Let $N \leq G$ be a normal subgroup and let $H=G / N$. Then $m_{\mathbb{H}}(G)=m_{\mathbb{H}}(H)$ if and only if $N$ is contained in all normal subgroups $N^{\prime}$ for which $G / N^{\prime}$ is a binary polyhedral group.

We will also require the following three lemmas. Let $\operatorname{Syl}_{p}(G)$ denote the isomorphism class of a Sylow $p$-subgroup of $G$ for $p$ prime.

Lemma 6.5. The proper quotients of $D_{2^{n}}$ and $Q_{2^{n}}$ are either $C_{2}$ or of the form $D_{2^{m}}$ for $2 \leq m \leq n-1$.

Lemma 6.6. If $1 \rightarrow N \rightarrow G \rightarrow H \rightarrow 1$ is an extension, then there is an extension of abstract groups $1 \rightarrow \operatorname{Syl}_{p}(N) \rightarrow \operatorname{Syl}_{p}(G) \rightarrow \operatorname{Syl}_{p}(H) \rightarrow 1$ for every prime $p$.

By combining these two lemmas with Theorem 6.1(iii), we see that any quotient $H$ of a group $G$ with periodic cohomology has $\operatorname{Syl}_{p}(H)$ cyclic for $p$ odd and $\operatorname{Syl}_{2}(H)$ cyclic, dihedral or quaternionic. 
Lemma 6.7. Suppose $G$ has periodic cohomology and that there exists non-trivial disjoint normal subgroups $N, N^{\prime} \leq G$ such that $H=G / N$ and $H^{\prime}=G / N^{\prime}$ are binary polyhedral groups. Then $G$ has a quotient of the form $Q_{4 n}$ for some $n \geq 6$. Furthermore $H$ and $H^{\prime}$ are not of the form $Q_{2^{n}}$ for any $n \geq 3, \widetilde{T}, \widetilde{O}$ or $\widetilde{I}$.

Proof. Suppose for contradiction that $H$ and $H^{\prime}$ are not of the form $Q_{4 n}$ for $n \geq 6$. Therefore $H$ and $H^{\prime}$ are each of the form $Q_{8}, Q_{12}, Q_{16}, Q_{20}, \widetilde{T}, \widetilde{O}$ or $\widetilde{I}$.

Since $N$ and $N^{\prime}$ are disjoint, $N \times N^{\prime} \leq G$ is a normal subgroup and so $H$ and $H^{\prime}$ have a common quotient $G /\left(N \times N^{\prime}\right) \cong H / N^{\prime} \cong H^{\prime} / N$. Furthermore, since $G$ has periodic cohomology, Theorem 6.1 (ii) implies that $|N|$ and $\left|N^{\prime}\right|$ are coprime. The remainder of the proof will be split into two cases.

First suppose that $\operatorname{Syl}_{2}(G)$ is cyclic. By Lemma 6.6 $\operatorname{Syl}_{2}(H)$ and $\operatorname{Syl}_{2}\left(H^{\prime}\right)$ must be quotients of $\operatorname{Syl}_{2}(G)$ and so are also cyclic. This implies $H$ and $H^{\prime}$ are each of the form $Q_{12}$ or $Q_{20}$. Since $|N|$ and $\left|N^{\prime}\right|$ are non-trivial and coprime, $|H| \neq\left|H^{\prime}\right|$ and so we can assume that $H=Q_{12}$ and $H^{\prime}=Q_{20}$. These groups have common quotients $1, C_{2}$ and $C_{4}$ and the restriction that $|N|$ and $\left|N^{\prime}\right|$ be coprime implies that $N=C_{5}$ and $N^{\prime}=C_{3}$. This implies, for example, that $G / C_{3} \cong Q_{20}$. Since $(3,20)=1$, this extension must split and so $G \cong C_{3} \rtimes_{\varphi} Q_{20}$ for some map $\varphi: Q_{20} \rightarrow \operatorname{Aut}\left(C_{3}\right) \cong C_{2}$. If $\varphi=1$, then $G \cong Q_{20} \times C_{3}$ which does not have $Q_{12}$ as a quotient. The only other option is that $\varphi$ is the quotient by $C_{10}$ which implies that $G \cong Q_{60}$.

Now suppose that $\operatorname{Syl}_{2}(G)=Q_{2^{n}}$ for some $n \geq 3$. Similarly Lemma 6.6 implies that $\operatorname{Syl}_{2}(H)$ and $\operatorname{Syl}_{2}\left(H^{\prime}\right)$ are quotients of $Q_{2^{n}}$. Since 4||$H|,| H^{\prime} \mid$ we can deduce, by Lemma 6.5. that $\operatorname{Syl}_{2}(H), \operatorname{Syl}_{2}\left(H^{\prime}\right)=Q_{2^{n}}$ and so $|N|,\left|N^{\prime}\right|$ are odd. Now $H$ and $H^{\prime}$ are each of the form $Q_{8}, Q_{16}, \widetilde{T}, \widetilde{O}$ or $\widetilde{I}$ and it is easy to verify that these groups have no non-trivial normal subgroups of odd order, which is a contradiction.

For the last part note that, if $H$ or $H^{\prime}$ were of the form $Q_{2^{n}}, \widetilde{T}, \widetilde{O}$ or $\widetilde{I}$, then we can get a contradiction using the same argument in the previous paragraph.

Proof of Theorem 6.3. Since $G$ does not satisfy the Eichler condition, there exists a binary polyhedral quotient $H=G / N$ which we can pick to have maximal order. Since $m_{\mathbb{H}}(G) \neq m_{\mathbb{H}}(H)$, Proposition 6.4 implies that there exists a binary polyhedral quotient $H^{\prime}=G / N^{\prime}$ for which $N \nsubseteq N^{\prime}$, and $N^{\prime} \nsubseteq N$ also by maximality of $|H|$. Now $G$ has quotient $\widehat{G}=G /\left(N \cap N^{\prime}\right)$ which has $H=G / K$ and $H^{\prime}=G / K^{\prime}$ for $K=N /\left(N \cap N^{\prime}\right)$ and $K^{\prime}=N^{\prime} /\left(N \cap N^{\prime}\right)$ disjoint normal subgroups, by the third isomorphism theorem. In addition, $K$ and $K^{\prime}$ are non-trivial since $N \cap N^{\prime} \neq N, N^{\prime}$.

If $\widehat{G}$ has periodic cohomology, then Lemma 6.7 implies that $G$ has a quotient of the form $Q_{4 n}$ for $n \geq 6$. Suppose that $\widehat{G}$ does not have periodic cohomology. Since $\operatorname{Syl}_{p}(\widehat{G})$ is a quotient of $\operatorname{Syl}_{p}(G)$ for all $p$ prime by Lemma 6.6, combining Lemma 6.5 and Theorem 6.1 (iii) shows that we must have $\operatorname{Syl}_{2}(\widehat{G})=D_{2^{n}}$ for some $n \geq 2$. Now Lemma 6.6 also implies that $\operatorname{Syl}_{2}(H)$ and $\operatorname{Syl}_{2}\left(H^{\prime}\right)$ are quotients of $D_{2^{n}}$. However, by Lemma 6.5, the only cyclic or generalised quaternionic quotients of $D_{2^{n}}$ are 1 or $C_{2}$ which contradicts the fact that 4||$H|,| H^{\prime} \mid$.

Recall that $m_{\mathbb{H}}\left(Q_{4 n}\right)=\lfloor n / 2\rfloor$ by [Jo03a, Section 12] and, using tables of real representations, it can be shown that $m_{\mathbb{H}}(\widetilde{T})=1, m_{\mathbb{H}}(\widetilde{O})=2$ and $m_{\mathbb{H}}(\widetilde{I})=2$.

Corollary 6.8. If $G$ has periodic cohomology, then the following are equivalent:

(i) $G$ has no quotient of the form $Q_{4 n}$ for $n \geq 6$.

(ii) $m_{\mathbb{H}}(G) \leq 2$. 
(iii) $G$ has a binary polyhedral quotient $H$ for which $m_{\mathbb{H}}(G)=m_{\mathbb{H}}(H) \leq 2$.

Proof. If $G$ has quotient $Q_{4 n}$ for $n \geq 6$, then $m_{\mathbb{H}}(G) \geq m_{\mathbb{H}}\left(Q_{4 n}\right) \geq 3$ by lifting the one dimensional quaternionic representations. Converesely, if $G$ has no quotient of the form $Q_{4 n}$ for $n \geq 6$, then Theorem 6.3 implies that either $m_{\mathbb{H}}(G)=0$ or $G$ has binary polyhedral quotient $H$ for which $m_{\mathbb{H}}(G)=m_{\mathbb{H}}(H)$. Since $H$ is not of the form $Q_{4 n}$ for $n \geq 6$, the results stated above imply that $m_{\mathbb{H}}(H) \leq 2$.

We have the following improvement to Theorem 6.3 in the case where $G$ has a quotient of the form $\widetilde{T}, \widetilde{O}$ or $\widetilde{I}$.

Proposition 6.9. If $G$ has periodic cohomology and has quotient $H=\widetilde{T}, \widetilde{O}$ or $\widetilde{I}$, then $m_{\mathbb{H}}(G)=m_{\mathbb{H}}(H) \leq 2$. In particular, $G$ has no quotient of the form $Q_{4 n}$ for $n \geq 6$.

Proof. If $m_{\mathbb{H}}(G) \neq m_{\mathbb{H}}(H)$, then Proposition 6.2 implies that there exists a binary polyhedral quotient $H^{\prime}=G / N^{\prime}$ for which $N \nsubseteq N^{\prime}$. Since $\widetilde{T}, \widetilde{O}$ and $\widetilde{I}$ have no proper quotients which are binary polyhedral groups, we must also have $N^{\prime} \nsubseteq N$ and so the group $\widehat{G}=G /\left(N \cap N^{\prime}\right)$ satisfies the conditions of Lemma 6.7 provided $\widehat{G}$ has periodic cohomology. Note that $\operatorname{Syl}_{2}(\widehat{G})$ has quotient $\operatorname{Syl}_{2}(H)=Q_{8}$ or $Q_{16}$ by Lemma 6.6 and so $\operatorname{Syl}_{2}(\widehat{G})$ is not dihedral by Lemma 6.5. This implies that $\widehat{G}$ has periodic cohomology and Lemma 6.7 then contradicts the fact that $H$ is of the form $\widetilde{T}, \widetilde{O}$ or $\widetilde{I}$. The second part now follows by Corollary 6.8

We now recall the classification of groups with 4-periodic cohomology using the notation of Milnor [Mi57. This can also be found in [Jo03a, Chapter 7].

Throughout, we will write $C_{n} \rtimes_{(r)} C_{m}$ to denote the semi-direct product where the generator $x \in C_{m}$ acts on the generator $y \in C_{n}$ by $x y x^{-1}=y^{r}$ for some $r \in \mathbb{Z}$. We also assume each family contains $G \times C_{n}$ for any $G$ listed with $(n,|G|)=1$.

(i) $C_{n}$ for $n \geq 1$, the cyclic group of order $n$.

(ii) $)^{\prime} D_{4 n+2}$ for $n \geq 1$, the dihedral group of order $4 n+2$.

(iii) ${ }^{\prime} Q_{4 n}=\left\langle x, y \mid x^{n}=y^{2}, y x y^{-1}=x^{-1}\right\rangle$ for $n \geq 2$ and $\widetilde{T}, \widetilde{O}, \widetilde{I}$.

(iv) $D\left(2^{n}, m\right)=C_{m} \rtimes_{(-1)} C_{2^{n}}$ for $n \geq 3$ and $m \geq 3$ odd.

$(\mathrm{v})^{\prime} P_{8 \cdot 3^{n}}^{\prime}=Q_{8} \rtimes_{\varphi} C_{3^{n}}$ for $n \geq 2$, where $\varphi: C_{3^{n}} \rightarrow$ Aut $Q_{8}$ sends the generator $z \in C_{3^{n}}$ to $\varphi(z): x \mapsto y, y \mapsto x y$.

(vi) $P_{48 n}^{\prime \prime}=C_{n} \cdot \widetilde{O}$ for $n \geq 3$ odd, the not-necessary-split extension which has cyclic Sylow 3 -subgroup and has action $\widetilde{O} \rightarrow \widetilde{O} / \widetilde{T}=C_{2} \leq$ Aut $C_{n}$.

(vii) $^{\prime} Q\left(2^{n} a ; b, c\right)=\left(C_{a} \times C_{b} \times C_{c}\right) \rtimes_{\varphi} Q_{2^{n}}$ for $n \geq 3$ and $a, b, c \geq 1$ odd coprime with $b>c$. If $C_{a}=\langle p\rangle, C_{b}=\langle q\rangle$ and $C_{c}=\langle r\rangle$, then the action is given by $\varphi(x): p \mapsto p^{-1}, q \mapsto q^{-1}, r \mapsto r \quad \varphi(y): p \mapsto p^{-1}, q \mapsto q, r \mapsto r^{-1}$.

We will now prove the following, which was stated in the introduction.

Theorem 6.10. The groups $G$ with 4-periodic cohomology for which $m_{\mathbb{H}}(G) \leq 2$ are as follows, where each family contains $G \times C_{n}$ for any $G$ listed with $(n,|G|)=1$.

(i) $C_{n}$ for $n \geq 1$.

(ii) $D_{4 n+2}$ for $n \geq 1$.

(iii) $Q_{8}, Q_{12}, Q_{16}, Q_{20}, \widetilde{T}, \widetilde{O}, \widetilde{I}$.

(iv) $D\left(2^{n}, 3\right), D\left(2^{n}, 5\right)$ for $n \geq 3$.

(v) $P_{8 \cdot 3^{n}}^{\prime}$ for $n \geq 2$.

(vi) $P_{48 n}^{\prime \prime}$ for $n \geq 3$ odd. 
(vii) $Q(16 ; m, n)$ for $m>n \geq 1$ odd coprime.

Proof. First note that we can ignore the groups of the form $G \times C_{n}$ for $G$ listed and $(n,|G|)=1$ since $m_{\mathbb{H}}\left(G \times C_{n}\right)=m_{\mathbb{H}}(G)$ in these cases.

It can be shown that the groups in (i) ${ }^{\prime}$, (ii) ${ }^{\prime}$ satisfy the Eichler condition [Jo03a, Section 12]. For the groups $G$ in (iii)', we use that $m_{\mathbb{H}}\left(Q_{4 n}\right)=\lfloor n / 2\rfloor, m_{\mathbb{H}}(\widetilde{T})=1$, $m_{\mathbb{H}}(\widetilde{O})=2$ and $m_{\mathbb{H}}(\widetilde{I})=2$ as mentioned previously.

In case (iv) ${ }^{\prime}$, suppose $G$ has a binary polyhedral quotient $H$. Explicit computation shows that $Z(H)=C_{2}$ and so the quotient map $f: G \rightarrow H$ must have $f(Z(G)) \subseteq Z(H)=C_{2}$. If $x \in C_{m}$ and $y \in C_{2^{n}}$ are generators, it is easy to see that $Z\left(D\left(2^{n}, m\right)\right)=\left\langle y^{2}\right\rangle=C_{2^{n-1}}$ which has index two subgroup $N=\left\langle y^{4}\right\rangle$. Hence $f$ factors through $G /\left\langle y^{4}\right\rangle=C_{m} \rtimes_{(-1)} C_{4}=Q_{4 m}$. By Proposition 6.4, we have that $m_{\mathbb{H}}(G)=m_{\mathbb{H}}\left(Q_{4 m}\right)=(m-1) / 2$ since $m$ is odd and so $m_{\mathbb{H}}(G) \leq 2$ if and only if $m=3$ or 5 and any $n \geq 3$.

The groups in $(\mathrm{v})^{\prime}$ all have quotient $\widetilde{T}$ and so $m_{\mathbb{H}}\left(P_{8 \cdot 3^{n}}^{\prime}\right)=1$ by Proposition 6.9 Similarly the groups in $(\text { vi })^{\prime}$ have quotient $\widetilde{O}$ and so $m_{\mathbb{H}}\left(P_{48 n}^{\prime \prime}\right)=2$. For the groups in (vii)', suppose $G=Q\left(2^{k} a ; b, c\right)$ has $m_{\mathbb{H}}(G) \leq 2$ for $a, b, c \geq 1$ odd coprime with $b>c$. Recall that, by Corollary 6.8, $m_{\mathbb{H}}(G) \leq 2$ if and only if $G$ has no quotient of the form $Q_{4 n}$ for $n \geq 6$. Since $G$ has quotient $Q_{2^{k} a}$ for $k \geq 3$, this implies that $a=1$ and $k=3$ or 4 . If $k=3$, then it is easy to see that $G \cong Q(8 c ; 1, b) \cong Q(8 b ; c, 1)$ and so has quotients $Q_{8 b}$ and $Q_{8 c}$. Hence $b=c=1$ which contradicts $b>c$.

Now suppose $k=4$, which we write as $G=Q(16 ; m, n)=\left(C_{n} \times C_{m}\right) \rtimes Q_{16}$ for $m>n \geq 1$ odd coprime. If $N^{\prime}=C_{n} \times C_{m}$, then $Q_{16}=G / N^{\prime}$. If $m_{\mathbb{H}}(G) \neq 2$, then Proposition 6.4 implies that $G$ has another binary polyhedral quotient $H=G / N$ such that $N^{\prime} \nsubseteq N$. If $\widehat{G}=G /\left(N \cap N^{\prime}\right)$, then $\operatorname{Syl}_{2}(\widehat{G})$ has quotient $Q_{16}$ by 6.6 which implies that $\operatorname{Syl}_{2}(\widehat{G})$ is not dihedral and so $\widehat{G}$ has periodic cohomology. If $N \nsubseteq N^{\prime}$, we could then apply Lemma 6.7 to get a contradiction since $Q_{16}=G / N^{\prime}$.

Hence we can assume that $N \subseteq N^{\prime}=C_{n} \times C_{m}=C_{n m}$ and so $N$ is of the form $C_{n^{\prime}} \times C_{m^{\prime}}$ for $n^{\prime} \leq n$ and $m^{\prime} \leq m$. It is easy to see that

$$
H=G / N=\left(C_{a} \times C_{b}\right) \rtimes_{\varphi^{\prime}} Q_{16}=Q(16 ; a, b)
$$

where $a=n / n^{\prime}$ and $b=n / n^{\prime}$. It is then a straightforward exercise to check that this is not a binary polyhedral group unless $a=b=1$. This implies that $N=N^{\prime}$ which contradicts the fact that $N^{\prime} \nsubseteq N$.

Remark 6.11. For the groups in the list, the groups in (i)', (ii) $)^{\prime}$ have $m_{\mathbb{H}}(G)=0$, and the groups $Q_{8} \times C_{n}, Q_{12} \times C_{n}$ and $\widetilde{T} \times C_{n}$ from (iii) $)^{\prime}$ and the groups in (v)' all have $m_{\mathbb{H}}(G)=1$. All other groups in the list have $m_{\mathbb{H}}(G)=2$.

We conclude this section by noting the following which we will use in Section 7

Corollary 6.12. Suppose $G$ has 4-periodic cohomology and $m_{\mathbb{H}}(G) \geq 3$. Then either $G$ has a quotient $Q_{4 n}$ for some $n \geq 7$ or $G \cong Q_{24} \times C_{n}$ where $(n, 24)=1$.

Proof. If $m_{\mathbb{H}}(G) \geq 3$ and $G$ does not have a quotient $Q_{4 n}$ for $n \geq 7$, then Theorem 6.3 implies that $G$ has a quotient $Q_{24}$. This rules out the groups in (i)', (ii)', (iv)', $(\mathrm{v})^{\prime}$ and (vi) ${ }^{\prime}$ by the proof of Theorem 6.10. If $G$ is in $(\mathrm{vii})^{\prime}$, then $G=Q\left(2^{k} a ; b, c\right) \times$ $C_{n}$ for $k \geq 3$ and $a, b, c, n \geq 1$ odd coprime with $b>c$. Since $G$ has no quotient $Q_{4 n}$ for $n \geq 7$, we must have that $k=3$. Since $Q(8 a ; b, c) \cong Q(8 b ; c, a) \cong Q(8 c ; a, b), G$ has quotients $Q_{8 a}, Q_{8 b}$ and $Q_{8 c}$ and so $a, b, c \leq 3$ which is a contradiction. Hence $G$ is in (iii)' which implies that $G \cong Q_{24} \times C_{n}$ for some $n \geq 1$ with $(n, 24)=1$. 


\section{CANCEllation OVER GRoups With PeRiodic COHOMOlOGY}

The aim of this section will be to prove the following two cancellation theorems, the latter of which will complete the proof of Theorem $\mathrm{A}$.

Theorem 7.1. Let $G$ have periodic cohomology. Then $\mathbb{Z} G$ has SFC if and only if $m_{\mathbb{H}}(G) \leq 2$.

Theorem 7.2. Let $G$ have 4-periodic cohomology and let $P_{G}$ be a projective $\mathbb{Z} G$ module for which $\sigma_{4}(G)=\left[P_{G}\right] \in C(\mathbb{Z} G) / T_{G}$. Then $\left[P_{G}\right]$ has cancellation if and only if $m_{\mathbb{H}}(G) \leq 2$.

We will begin by proving Theorem 7.1. Firstly note that, if $G$ satisfies the Eichler condition, then Theorem 5.2 implies that $\mathbb{Z} G$ has SFC. In the case where $G$ does not satisfy the Eichler condition, we have the following result of the author:

Theorem 7.3 ([Ni21, Theorem B]). Suppose $G$ has a binary polyhedral quotient $H$ such that $m_{\mathbb{H}}(G)=m_{\mathbb{H}}(H)$. Then $\mathbb{Z} G$ has $\mathrm{SFC}$ if and only if $\mathbb{Z} H$ has $\mathrm{SFC}$.

This is a consequence of [Ni21, Theorem A], which itself can be obtained by specialising Theorem 5.1 to $\bar{P}=\mathbb{Z} G$ and combining with the following two results.

Theorem 7.4 ([MOV83, Theorems 7.15-7.18]). If $H=Q_{8}, Q_{12}, Q_{16}, Q_{20}, \widetilde{T}, \widetilde{O}, \widetilde{I}$, then the map $\mathbb{Z} H^{\times} \rightarrow K_{1}(\mathbb{Z} H)$ is surjective.

Theorem 7.5 ([Sw83, Theorem I]). If $G$ is a binary polyhedral group, then $\mathbb{Z} G$ has $\mathrm{SFC}$ if and only if $G$ is of the form $Q_{8}, Q_{12}, Q_{16}, Q_{20}, \widetilde{T}, \widetilde{O}, \widetilde{I}$.

The latter is a special case of Theorem 7.1 since these are the binary polyhedral groups for which $m_{\mathbb{H}}(G) \leq 2$.

Proof of Theorem 7.1. Suppose $\mathbb{Z} G$ has SFC. By combining Theorems 5.4 and 7.5, we get that $G$ has no quotient of the form $Q_{4 n}$ for $n \geq 6$. By Corollary 6.8, this implies that $m_{\mathbb{H}}(G) \leq 2$.

Suppose instead that $m_{\mathbb{H}}(G) \leq 2$. By Corollary 6.8 we get that $G$ has a binary polyhedral quotient $H$ for which $m_{\mathbb{H}}(G)=m_{\mathbb{H}}(H) \leq 2$. By Theorem 7.5 , this implies that $\mathbb{Z} H$ has SFC and so $\mathbb{Z} G$ has SFC by Theorem 7.3

We now turn to the proof of Theorem 7.2 . We require the following results:

Lemma 7.6 ( $\underline{\text { Sw83, }}$ Theorem I]). If $G=Q_{4 n}$ for $n \geq 7$, then $[P]$ has noncancellation for every projective $\mathbb{Z} G$-module $P$.

Lemma 7.7 ([CR90, Proposition 53.10]). Let $N \leq G$ be a normal subgroup. Then the induced map $-\otimes_{\mathbb{Z} N} \mathbb{Z}: T_{G} \rightarrow T_{G / N}$ is surjective.

The main tool in the proof of Theorem 7.2 will be the following lemma. This is the only place in this article that we will use Theorem 5.1 instead of Theorem 7.3 . which is strictly weaker and was already proven in $\mathrm{Ni21}$.

Lemma 7.8. Let $G$ have 4-periodic cohomology and let $P_{G}$ be a projective $\mathbb{Z} G$ module for which $\sigma_{4}(G)=\left[P_{G}\right] \in C(\mathbb{Z} G) / T_{G}$. Suppose there exists a normal subgroup $N \leq G$ for which $H=G / N$ is a binary polyhedral group, $m_{\mathbb{H}}(G)=m_{\mathbb{H}}(H) \leq 2$ and $\left[P_{G} \otimes_{\mathbb{Z} N} \mathbb{Z}\right] \in T_{H}$. Then $\left[P_{G}\right]$ has cancellation. 
Proof. It follows from Theorem 4.1 that the property that $\left[P_{G}\right]$ has cancellation is independent of the choice of representative $P_{G}$ for $\sigma_{4}(G)$. Therefore, it suffices to determine cancellation for any choice of $P_{G}$.

By Lemma 7.7, there exists $[P] \in T_{G}$ with $\left[P \otimes_{\mathbb{Z} N} \mathbb{Z}\right]=-\left[P_{G} \otimes_{\mathbb{Z N N}} \mathbb{Z}\right] \in C(\mathbb{Z} H)$. By [Sw60a, Theorem A], there exists pa rojective $\mathbb{Z} G$ module $P_{G}^{\prime}$ of rank one such that $P_{G} \oplus P \cong P_{G}^{\prime} \oplus \mathbb{Z} G^{r}$. This satisfies $\left[P_{G}^{\prime}\right]=\left[P_{G}\right] \in C(\mathbb{Z} G) / T_{G}$ and $\left[P_{G}^{\prime} \otimes_{\mathbb{Z} N} \mathbb{Z}\right]=0 \in C(\mathbb{Z} H)$. By Theorem 7.1, $\mathbb{Z} H$ has SFC and so $P_{G}^{\prime} \otimes_{\mathbb{Z} N} \mathbb{Z}=\mathbb{Z} H$.

By Theorem 7.4, we have that $\operatorname{Aut}(\mathbb{Z} H)=\mathbb{Z} H^{\times} \rightarrow K_{1}(\mathbb{Z} H)$. Hence the conditions of Theorem 5.1 are satisfies and so $\left[P_{G}^{\prime}\right]$ has cancellation.

It follows from results of J. A. Wolf Wo74, Chapter 6] that, if $G$ is in (i) ${ }^{\prime}$, (iii)', $(\text { iv })^{\prime},(\mathrm{v})^{\prime}$, then $G$ is a fixed-point free finite subgroups of $S O(4)$ and so is the fundamental group of a closed 3-manifold $M$. Since $C_{*}(\widetilde{M}) \in \operatorname{hProj}_{\mathbb{Z} G}^{4}(\mathbb{Z}, \mathbb{Z} ; 0)$, this implies that $\sigma_{4}(G)=0$ for these groups. This also holds for the groups in (ii)' (see [Jo03a, p236] or Wa79b, Section 12]).

Lemma 7.9. If $G$ is in $(\mathrm{i})^{\prime}-(\mathrm{v})^{\prime}$, then $\sigma_{4}(G)=0 \in C(\mathbb{Z} G) / T_{G}$.

The following lemma is standard.

Lemma 7.10. If $N \unlhd G$ and $N \leq H \leq G$, then there is a commutative diagram:

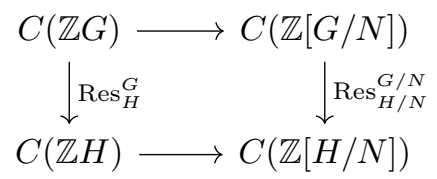

where the horizontal maps are induced by $-\otimes_{\mathbb{Z} N} \mathbb{Z}$.

Proof of Theorem 7.2. As in the proof of Lemma 7.8, it will suffice to prove that $\left[P_{G}\right]$ has cancellation or non-cancellation where $P_{G}$ is any representative for $\sigma_{4}(G)$.

To prove the forward direction, suppose $m_{\mathbb{H}}(G) \geq 3$. By Corollary 6.12, we know that $G$ either has a quotient of the form $Q_{4 n}$ for $n \geq 7$ or $G \cong Q_{24} \times C_{n}$ where $(n, 24)=1$. If $G$ has a quotient $Q_{4 n}=G / N$ for $n \geq 7$ and $P=P_{G} \otimes_{\mathbb{Z} N} \mathbb{Z}$, then $[P]$ has non-cancellation by Theorem $\left[7.6\right.$ and so $\left[P_{G}\right]$ has non-cancellation also by Theorem 5.4. If $G=Q_{24} \times C_{n}$, then $\sigma_{4}(G)=0$ by Lemma 7.9 and so we can take $\left[P_{G}\right]=[\mathbb{Z} G]$. Note that $\left[\mathbb{Z} Q_{24}\right]$ non-cancellation by Theorem 7.5 and so $[\mathbb{Z} G]$ has non-cancellation by Theorem 5.4 .

For the converse, suppose $m_{\mathbb{H}}(G) \leq 2$. If $\sigma_{4}(G)=0$, then we can take $\left[P_{G}\right]=$ $[\mathbb{Z} G]$ which has cancellation by Theorem 7.1 . If $\sigma_{4}(G) \neq 0$, then Lemma 7.9 implies that $G$ is one of the groups in (vi) or (vii). If $G$ is in (vii), then $G=Q(16 ; m, n) \times C_{r}$ for $m, n$ odd coprime and $(r, 16 m n)=1$. By Theorem 6.10, the quotient $Q_{16}=$ $G / N$ has $m_{\mathbb{H}}(G)=m_{\mathbb{H}}\left(Q_{16}\right)=2$. It follows from [Sw83, Theorems III, VI] that $C\left(\mathbb{Z} Q_{16}\right)=T_{Q_{16}}$ and so $\left[P_{G} \otimes_{\mathbb{Z} N} \mathbb{Z}\right] \in T_{Q_{16}}$ for all projective $\mathbb{Z} G$ modules $P_{G}$ for which $\sigma_{4}(G)=\left[P_{G}\right] \in C(\mathbb{Z} G) / T_{G}$. Hence $\left[P_{G}\right]$ has cancellation by Lemma 7.8 .

If $G$ is in (vi), then $G=P_{48 n}^{\prime \prime} \times C_{m}$ for $n \geq 3$ odd and $m \geq 1$ with $(48 n, m)=1$. Theorem 6.10 implies that the quotient $\widetilde{O}=G / N$ has $m_{\mathbb{H}}(G)=m_{\mathbb{H}}(\widetilde{O})=2$ where $N=C_{n} \times C_{m}$. It is proven in [Sw83, Theorem 14.1] that the restriction map Res : $C(\mathbb{Z} \widetilde{O}) \rightarrow C\left(\mathbb{Z} Q_{16}\right) \oplus C\left(\mathbb{Z} Q_{12}\right)$ is bijective. This restricts to an injection Res $\left.\right|_{\widetilde{O}}: T_{\widetilde{O}} \rightarrow T_{Q_{16}} \oplus T_{Q_{12}}$ which is necessarily bijective since $T_{\widetilde{O}} \cong \mathbb{Z} / 2, T_{Q_{16}} \cong$ $\mathbb{Z} / 2$ and $T_{Q_{12}}=0\left[\right.$ Sw83, Theorem VI]. Since $C\left(\mathbb{Z} Q_{16}\right)=T_{Q_{16}}$, a class $[P] \in C(\mathbb{Z} \widetilde{O})$ is contained in $T_{\widetilde{O}}$ if and only if $\operatorname{Res}_{Q_{12}}^{\widetilde{O}}(P)=0 \in C\left(\mathbb{Z} Q_{12}\right)$. 
Note that $G$ has a (hyperelementary) subgroup $H=Q_{12 n} \times C_{m}$ which contains $N$ and has $Q_{12}=H / N$. By Lemma 7.10, we now have a commutative diagram:

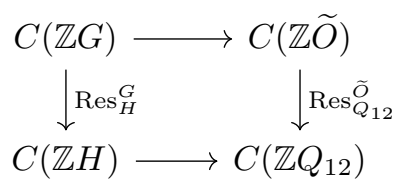

Let $P_{G}$ be such that $\sigma_{4}(G)=\left[P_{G}\right] \in C(\mathbb{Z} G) / T_{G}$. If $P_{H}=\operatorname{Res}_{H}^{G}\left(P_{G}\right)$, then it is straightforward to see that $\sigma_{4}(H)=\left[P_{H}\right] \in C(\mathbb{Z} H) / T_{H}$. Since $H$ is in (iii), Lemma 7.9 implies that $\sigma_{4}(H)=0$ and so $\left[P_{H}\right] \in T_{H}$. This implies that $\left[P_{H} \otimes_{\mathbb{Z} N}\right.$ $\mathbb{Z}]=0$ since it is contained in $T_{Q_{12}}=0$. Hence $\operatorname{Res}_{H}^{G}\left(P_{G}\right) \otimes_{\mathbb{Z N}} \mathbb{Z}=0 \in C\left(\mathbb{Z} Q_{12}\right)$ and so, as the diagram above commutes, we have $\operatorname{Res}_{Q_{12}}^{\widetilde{O}}\left(P_{G} \otimes_{\mathbb{Z} N} \mathbb{Z}\right)=0 \in C\left(\mathbb{Z} Q_{12}\right)$. Hence $\left[P_{G} \otimes_{\mathbb{Z} N} \mathbb{Z}\right] \in T_{\widetilde{O}}$ which implies that $\left[P_{G}\right]$ has cancellation by Lemma 7.8 .

Proof of Theorem $A$. Since D2 $(G)$ is connected, finite D2 complexes $X$ with $\pi_{1}(X)=$ $G$ are determined up to homotopy by $\chi(X)$ if and only if $\mathrm{D} 2(G)$ has cancellation.

Let $P_{G}$ be such that $\sigma_{4}(G)=\left[P_{G}\right] \in C(\mathbb{Z} G) / T_{G}$. By combining Theorems 2.1 and 4.1, we get that there is an isomorphism of graded trees

$$
\Psi \circ \widetilde{C}_{*}: \mathrm{D} 2(G) \rightarrow\left[P_{G}\right]
$$

and so D2 $(G)$ has cancellation if and only if $\left[P_{G}\right]$ has cancellation. By Theorem 7.2 $\left[P_{G}\right]$ has cancellation if and only if $m_{\mathbb{H}}(G) \leq 2$, which completes the proof.

\section{BALANCED PRESENTATIONS AND THE D2 PROBLEM}

We will now show how the following can be deduced from Theorem A,

Corollary B, Let $G$ have 4-periodic cohomology. Then:

(i) If $G$ has the $\mathrm{D} 2$ property, then $G$ has a balanced presentation.

(ii) If $G$ has a balanced presentation and $m_{\mathbb{H}}(G) \leq 2$, then $G$ has the D2 property.

Proof. Recall that, as discussed at the end of Section 2 , $G$ has the D2 property if and only if the induced map $\Psi \circ \widetilde{C}_{*}: \operatorname{PHT}(G, 2) \rightarrow\left[P_{G}\right]$ is bijective.

Suppose $G$ has the D2 property. Then $\Psi \circ \widetilde{C}_{*}$ is bijective and, since $\left[P_{G}\right]$ contains a projective $\mathbb{Z} G$ module of rank one, there must exist $(X, \rho) \in P H T(G, 2)$ such that $\chi(X)=1$. If $\mathcal{P}$ is a presentation such that $X \simeq X_{\mathcal{P}}$, then $\operatorname{def}(\mathcal{P})=1-\chi(X)=0$ and so $\mathcal{P}$ is a balanced presentation as required.

Suppose instead that $m_{\mathbb{H}}(G) \leq 2$ and that $G$ has a balanced presentation $\mathcal{P}$. By Theorem $\mathrm{A}\left[P_{G}\right]$ has cancellation and so $\left[P_{G}\right]=\left\{P_{0} \oplus \mathbb{Z} G^{r}: r \geq 0\right\}$ for some projective $P_{0}$ of rank one. Let $P_{1}=\Psi\left(\widetilde{C}_{*}\left(X_{\mathcal{P}}\right)\right)$, which has $\operatorname{rank}\left(P_{1}\right)=1$ since $\chi\left(X_{\mathcal{P}}\right)=1$. Since $P_{1} \in[P]$, this implies that $P_{1} \cong P_{0}$. In particular, for all $r \geq 0$, we have $\Psi\left(\widetilde{C}_{*}\left(X_{\mathcal{P}} \vee r S^{2}\right)\right) \cong P_{0} \oplus \mathbb{Z} G^{r}$ and so $\Psi \circ \widetilde{C}_{*}$ is surjective. Since $\Psi \circ \widetilde{C}_{*}$ is injective, this implies that it is bijective and so $G$ has the D2 property.

Corollary C, Let $X$ be a finite Poincaré 3-complex with $G=\pi_{1}(X)$ finite. Then:

(i) If $X$ has a cell structure with a single 3 -cell, then $G$ has a balanced presentation.

(ii) If $G$ has a balanced presentation and $m_{\mathbb{H}}(G) \leq 2$, then $X$ has a cell structure with a single 3 -cell. 
Proof. Let $X$ be a finite Poincaré 3-complex with $G=\pi_{1}(X)$ finite. By Corollary 3.4. $G$ has 4-periodic cohomology.

Suppose $G$ has a cell structure with a single 3 -cell $e^{3}$. Then $X=X^{(2)} \cup e^{3}$, where the 2-skeleton $X^{(2)}$ is a finite 2-complex. It follows from Poincaré duality that $\chi(X)=0$ and so $\chi\left(X^{(2)}\right)=1$. If $\mathcal{P}$ is a presentation such that $X^{(2)} \simeq X_{\mathcal{P}}$, then $\operatorname{def}(\mathcal{P})=1-\chi\left(X^{(2)}\right)=0$ and so $\mathcal{P}$ is a balanced presentation as required.

Suppose instead that $m_{\mathbb{H}}(G) \leq 2$ and that $G$ has a balanced presentation. By Corollary B, $G$ has the D2 property. By Wa67, Theorem 2.4], there exists a finite D2 complex $K$, a map $\alpha: S^{2} \rightarrow K$ and a homotopy equivalence $X \rightarrow K \cup_{\alpha} e^{3}$. Since $G$ has the D2 property, there is a homotopy equivalence $h: K \rightarrow K_{0}$ for some finite 2-complex $K_{0}$. By combining these maps, we obtain a homotopy equivalence $X \rightarrow$ $K_{0} \cup_{h \circ \alpha} e^{3}$ which gives a cell structure for $X$ with a single 3-cell as required.

For the remainder of this section, we will discuss the implications of Corollaries $\mathrm{B}$ and $\mathrm{C}$ on the D2 problem over groups with 4-periodic cohomology.

8.1. Balanced presentations for groups with periodic cohomology. If $G$ has periodic cohomology, then $H_{2}(G ; \mathbb{Z})=0$ (see, for example, Sw71). In particular, by Corollary $\mathrm{B}$, the groups $G$ with 4-periodic cohomology are either counterexamples to the D2 problem or give a new supply of groups with efficient presentations.

This gives some response to comments made by L. G. Kovács [Ko94, p212] and J. Harlander [Har98, p167] on the scarcity of efficient finite groups. In contrast, there is currently no example known to contradict the following conjecture:

Conjecture 8.1. If $G$ has periodic cohomology, then $G$ has a balanced presentation.

By [Wo74, Chapter 6], the groups in $(\mathrm{i})^{\prime},(\mathrm{iii})^{\prime},(\mathrm{iv})^{\prime},(\mathrm{v})^{\prime}$ are all finite 3 -manifold groups which are well-known to have balanced presentations. This follows, for example, from Corollary [C] since 3-manifolds have cell structures with a single 3cell. The groups in (ii)' also have balanced presentations (see [Jo03a, p236]).

Proposition 8.2. If $G$ is in (i)'-(v)', then $G$ has a balanced presentation.

We have not been able to find balanced presentations for any of the groups in (vi), but have succeeded for the following groups in (vii). These groups overlap in the case where $k=n=1$ with the groups in [Ne89, Theorem 3.1].

Proposition 8.3. If $n \geq 3$ and $a, b, k \geq 1$ odd coprime, then:

$$
Q\left(2^{n} a ; b, 1\right) \times C_{k} \cong\left\langle x, y \mid y^{k} x^{b} y^{k}=x^{b}, x y x=y^{2^{n-2} a-1}\right\rangle .
$$

By combining Propositions 8.2 and 8.3 with Corollary $\mathrm{B}$ we obtain:

Theorem 8.4. Suppose $G$ is in (i)-(v) or has the form $Q(16 ; n, 1) \times C_{k}$ for some $n, k \geq 1$ odd coprime. Then $G$ has the $\mathrm{D} 2$ property.

Note that not all of these groups have free period 4 ; an example is $Q(16 ; 3,1)$ Da82. The simplest groups that we have not been able to find balanced presentations for are $P_{48.3}^{\prime \prime}$ and $Q(16 ; 3,5)$. The following is therefore of practical interest:

Question 8.5. Do $P_{48 \cdot 3}^{\prime \prime}$ or $Q(16 ; 3,5)$ have balanced presentations?

These correspond to the groups $G_{144}^{31}, G_{240}^{22}$ in GAP's the Small Groups Library. 
8.2. Potential counterexamples to the $\mathbf{D} 2$ problem. Recall that a $\mathbb{Z} G$ module $M$ has the Swan property (or is a 'Swan module') if

$$
d_{\mathbb{Z} G}(M)=\max _{p|| G \mid} d_{\mathbb{Z}_{p} G}\left(M \otimes \mathbb{Z}_{p}\right)
$$

where $d_{R}(\cdot)$ is the number of $R$-module generators.

In 1977, J. M. Cohen proposed the group $Q_{32}$ as a counterexample to the D2 problem [Co77, Section 4]. More generally, he conjectured the following.

Conjecture 8.6 (Wa79a, Problem D3]). Let $X$ be a finite D2 complex. Then $X$ is homotopy equivalent to a finite 2-complex if and only if $\pi_{2}(X)$ has the Swan property or $\pi_{1}(X)$ is infinite.

The following was proved by Cohen as a consequence of [Co78, Theorem 3], where $I$ is the augmentation ideal. This gives another reason why the D2 property for groups with 4-periodic cohomology is of particular interest.

Proposition 8.7 ([Co77, p415]). Let $X$ be a finite D2 complex such that $\pi_{2}(X)$ does not have the Swan property and $G=\pi_{1}(X)$ is finite. Then:

(i) $G$ has free period 4 .

(ii) $\chi(X)=1$.

(iii) $\pi_{2}(X)$ is non-cyclic, i.e. $\pi_{2}(X) \neq I^{*}$.

We will now show the following as an application of Theorem $\mathrm{A}$. Recall that $\mathbb{Z} G$ modules $A$ and $B$ are Aut $(G)$-isomorphic if there exists a bijection $\varphi: A \rightarrow B$ such that, for some $\theta \in \operatorname{Aut}(G)$, we have $\varphi(g \cdot x)=\theta(x) \cdot \varphi(x)$ for all $x \in A$.

Corollary 8.8. Suppose the "if" part of Conjecture 8.6 holds. If G does not have the $\mathrm{D} 2$ property, then $G$ has free period 4 and $m_{\mathbb{H}}(G) \geq 3$.

Proof. By Proposition 8.7, $G$ has free period 4 and there exists a finite D2 complex $X$ with $\pi_{1}(X)=G, \chi(X)=1$ and $\pi_{2}(X) \neq I^{*}$. Recall that, in Proposition 4.2, we constructed a finite D2 complex $X_{G}$ with $\pi_{1}\left(X_{G}\right)=G$ and $\chi\left(X_{G}\right)=1$ whenever $G$ has 4-periodic cohomology. Since $G$ has free period 4, we can take $J=I^{*}$ in the proof of Proposition 4.2 which gives that $\pi_{2}\left(X_{G}\right) \cong I^{*}$. In particular, $X \vee r S^{2} \simeq$ $X_{G} \vee r S^{2}$ for some $r \geq 1$. If $X \simeq X_{G}$, then $\pi_{2}(X)$ and $\pi_{2}\left(X_{G}\right)$ would be $\operatorname{Aut}(G)$ isomorphic which is a contradiction since $\pi_{2}(X)$ is non-cyclic and $\pi_{2}\left(X_{G}\right) \cong I^{*} \cong$ $\mathbb{Z} G / \Sigma$. Hence $\mathrm{D} 2(G)$ has non-cancellation and so $m_{\mathbb{H}}(G) \geq 3$ by Theorem $\mathrm{A}$

We say that two presentations $\mathcal{P}$ and $\mathcal{Q}$ for a group $G$ are exotic if $X_{\mathcal{P}} \neq X_{\mathcal{Q}}$ and $\operatorname{def}(\mathcal{P})=\operatorname{def}(\mathcal{Q})$ or, equivalently, if $X_{\mathcal{P}} \vee r S^{2} \simeq X_{\mathcal{Q}} \vee r S^{2}$ for some $r \geq 0$. The following was shown recently by Mannan and Popiel [MP21. This is, to date, the only known example of an exotic presentation for a finite non-abelian group.

Theorem 8.9 ([MP21, Theorem A]). The quaternion group $Q_{28}$ has presentations

$$
\mathcal{P}_{1}=\left\langle x, y \mid x^{7}=y^{2}, x y x=y\right\rangle, \quad \mathcal{P}_{2}=\left\langle x, \mid x^{7}=y^{2}, y^{-1} x y x^{2}=x^{3} y^{-1} x^{2} y\right\rangle
$$

such that $\pi_{2}\left(X_{\mathcal{P}_{1}}\right) \neq \pi_{2}\left(X_{\mathcal{P}_{2}}\right)$ are not $\operatorname{Aut}\left(Q_{28}\right)$-isomorphic. Hence $X_{\mathcal{P}_{1}} \not 千 X_{\mathcal{P}_{2}}$.

We now proceed to point out the following two consequences.

Theorem 8.10. The "only if" part of Conjecture 8.6 is false. 
Proof. It is noted after the proof of [MP21, Theorem A] that $d_{\mathbb{Z} Q_{28}}\left(\pi_{2}\left(X_{\mathcal{P}_{1}}\right)\right)=1$ and $d_{\mathbb{Z} Q_{28}}\left(\pi_{2}\left(X_{\mathcal{P}_{1}}\right)\right) \neq 1$. However, $\pi_{2}\left(X_{\mathcal{P}_{1}}\right) \oplus \mathbb{Z} G^{r} \cong \pi_{2}\left(X_{\mathcal{P}_{2}}\right) \oplus \mathbb{Z} G^{r}$ for some $r \geq 0$ and it follows that $\pi_{2}\left(X_{\mathcal{P}_{1}}\right) \otimes \mathbb{Z}_{p} \cong \pi_{2}\left(X_{\mathcal{P}_{2}}\right) \otimes \mathbb{Z}_{p}$ for $p|| G \mid$ prime since $\mathbb{Z}_{p} G$ is semisimple by Maschke's theorem. This implies that $d_{\mathbb{Z}_{p} G}\left(\pi_{2}\left(X_{\mathcal{P}_{2}}\right) \otimes \mathbb{Z}_{p}\right)=1$ for all $p|| G \mid$ and so $\pi_{2}\left(X_{\mathcal{P}_{2}}\right)$ does not have the Swan property. This contradicts the "only if" direction of Conjecture 8.6 since $X_{\mathcal{P}_{2}}$ is a finite 2-complex.

Theorem 8.11. $Q_{28}$ has the D2 property and $m_{\mathbb{H}}\left(Q_{28}\right)=3$.

In BW05, this is proposed as a counterexample by F. R. Beyl and N. Waller and so this answers their question in the negative (see also [MR18, p23]).

Proof. Note that $Q_{28}$ is in (iii) and so has $\sigma_{4}\left(Q_{28}\right)=0$ by Lemma 7.9. Hence, as in the proof of Corollary B it suffices to show that the injective map of graded trees $\Psi \circ \widetilde{C}_{*}: \operatorname{PHT}\left(Q_{28}, 2\right) \rightarrow\left[\mathbb{Z} Q_{28}\right]$ is in fact bijective. By the discussion in Section 4 , $\operatorname{PHT}\left(Q_{28}, 2\right)$ and $\left[\mathbb{Z} Q_{28}\right]$ are both forks. By $[\mathrm{Sw} 83$, Theorem III], there are two rank one stably free $\mathbb{Z} Q_{28}$ modules and so $\left[\mathbb{Z} Q_{28}\right]$ has two vertices at the minimal level. By Theorem 8.9, $\operatorname{PHT}\left(Q_{28}, 2\right)$ has at least two vertices at the minimal level. Hence

the injective map $\Psi \circ \widetilde{C}_{*}$ must be bijective, and so $Q_{28}$ has the D2 property.

It should be possible to replicate this proof for other examples of groups with 4-periodic cohomology and $m_{\mathbb{H}}(G) \geq 3$. We expect that the quaternion groups $Q_{4 n}$ contain the main difficulties associated with the case $m_{\mathbb{H}}(G) \geq 3$, and so we ask:

Question 8.12. Does $Q_{4 n}$ have the D2 property for all $n \geq 2$ ?

The case $Q_{32}$ is of particular significance since it was the first proposed counterexample to the D2 problem Co77, p415].

\section{ACKNOWLEDGEMENTS}

I would like to thank my supervisor F. E. A. Johnson for many interesting conversations on the D2 problem and stable modules, W. H. Mannan for drawing my attention to his recent work on exotic presentations for $Q_{28}$ and Jonathan Hillman for many helpful comments. I am also indebted to an anonymous referee for their careful reading and many useful suggestions. In particular, they suggested I give an explicit form for the map $\Psi$ from Theorem 4.1, which can now be found in Proposition 4.12, This work was supported by the UK Engineering and Physical Sciences Research Council (EPSRC) grant EP/N509577/1.

\section{REFERENCES}

[BW05] F. R. Beyl, N. Waller, A stably free nonfree module and its relevance for homotopy classification, case $Q_{28}$, Algebr. Geom. Topol. 5 (2005), 899-910.

[Br78] W. J. Browning, Homotopy types of certain finite $C W$-complexes with finite fundamental group, PhD Thesis, Cornell University (1978).

[CE56] H. Cartan, S. Eilenberg, Homological algebra, Princeton Mathematics Series 19 (1956).

[Co77] J. M. Cohen, Complexes dominated by a 2-complex, Topology 16 (1977), 409-415.

[Co78] J. M. Cohen, On the number of generators of a module, J. Pure Appl. Algebra 12 (1978), no. $1,15-19$.

[CR90] C. W. Curtis, I. Reiner, Methods of representation theory: with applications to finite groups and orders, Volume 2, Wiley Classics Library 26 (1990).

[Da82] J. F. Davis, Evaluation of the Swan finiteness obstruction, PhD Thesis, Stanford University (1982). 
[Dy75] M. N. Dyer, On the 2-realizability of 2-types, Trans. Amer. Math. Soc. 204 (1975), 229243.

[Fr75] A. Fröhlich, Locally free modules over arithmetic orders, J. Reine Angew. Math. 274/275 (1975), 112-138.

[Ham19] I. Hambleton, Two remarks on Wall's D2 problem, Math. Proc. Cambridge Philos. Soc. 167 (2019), 361-368.

[Har98] J. Harlander, Some aspects of efficiency, Groups - Korea 98: Proceedings of the International Conference held at Pusan (1998).

[Ja68] H. Jacobinski, Genera and decompositions of lattices over orders, Acta Math. 121 (1968), $1-29$.

[Jo04] F. E. A. Johnson, Minimal 2-complexes and the D(2)-problem, Proc. Amer. Math. Soc. 132 (2004), 579-586.

[Jo03a] F. E. A. Johnson, Stable modules and the D(2)-problem, London Math. Soc. Lecture Note Ser. 301, Cambridge University Press (2003).

[Jo03b] F. E. A. Johnson, Stable modules and Wall's D(2)-problem, Comment. Math. Helv. 78 (2003), 18-44.

[Ko94] L. G. Kovács, Finite groups with trivial multiplicator and large deficiency, Groups - Korea 94: Proceedings of the International Conference held at Pusan (1994).

[MOV83] B. Magurn, R. Oliver, L. Vaserstein, Units in Whitehead groups of finite groups, J. of Algebra 84 (1983), 324-360.

[Ma09] W. H. Mannan, Realizing algebraic 2-complexes by cell complexes, Math. Proc. Cambridge Philos. Soc. 146 (3) (2009), 671-673.

[MP21] W. H. Mannan, T. Popiel, An exotic presentation of $Q_{28}$, Algebr. Geom. Topol., to appear, arXiv:1901.10786 (2021).

[MR18] W. Metzler, S. Rosebrock, Advances in two-dimensional homotopy and combinatorial group theory, London Math. Soc. Lecture Note Ser. 446, Cambridge University Press (2018).

[Mi85] R. J. Milgram, Evaluating the Swan finiteness obstruction for periodic groups, Algebraic and geometric topology (New Brunswick, N.J., 1983), Lecture Notes in Math. 1126, Springer, Berlin, (1985), 127-158.

[Mi71] J. Milnor, Introduction to algebraic K-theory, Ann. of Math. Stud. 72 (1971).

[Mi57] J. Milnor, Groups which act on $S^{n}$ without fixed points, Amer. J. Math. 79 (3) (1957), 623-630.

[Ne89] B. H. Neumann, Yet more on finite groups with few defining relations, Proceedings of the Singapore Group Theory Conference held at the National University of Singapore, June 8-9, 1987 (1989), 183-193.

[Ni21] J. Nicholson, A cancellation theorem for modules over integral group rings, Math. Proc. Cambridge Philos. Soc., to appear, arXiv:1807.00307 (2021).

[Ni20] J. Nicholson, Projective modules and the homotopy classification of $(G, n)$-complexes, arXiv:2004.04252 (2020)

[Ol65] P. Olum, Self-equivalences of pseudo-projective planes, Topology 4 (1965), 109-127.

[St68] J. R. Stallings, On torsion free groups with infinitely many ends, Ann. of Math. (2) 88 (1968), 312-334.

[Sw69] R. G. Swan, Groups of cohomological dimension one, J. of Algebra 12 (1969), 585-610.

[Sw71] R. G. Swan, Groups with no odd dimensional cohomology, J. of Algebra 17 (1971), 401403.

[Sw70] R. G. Swan, K-theory of finite groups and orders, Lecture Notes in Math. 149, Springer (1970).

[Sw65] R. G. Swan, Minimal resolutions for finite groups, Topology 4 (1965), 193-220.

[Sw60a] R. G. Swan, Induced representations and projective modules, Ann. of Math. (2) 71 (1960), $552-578$.

[Sw60b] R. G. Swan, Periodic resolutions for finite groups, Ann. of Math. (2) 72 (1960), 267-291.

[Sw83] R. G. Swan, Projective modules over binary polyhedral groups, J. Reine Angew. Math. 342 (1983), 66-172.

[Sw62] R. G. Swan, Projective modules over group rings and maximal orders, Ann. of Math. (2) 76 (1962), 55-61.

[Sw80] R. G. Swan, Strong approximation and locally free modules, Ring Theory and Algebra III, proceedings of the third Oklahoma Conference 3 (1980), 153-223.

[Wa65] C. T. C. Wall, Finiteness conditions for CW complexes, Ann. of Math. 81 (1965), 56-69. 
[Wa66] C. T. C. Wall, Finiteness conditions for CW complexes. II, Proc. Roy. Soc. Ser. A 295 (1966), 129-139.

[Wa79a] C. T. C. Wall, Homological group theory, London Math. Soc. Lecture Note Ser. 35, Cambridge University Press (1979).

[Wa79b] C. T. C. Wall, Periodic projective resolutions, Proc. London Math. Soc. (3) 39 (1979), 509-553.

[Wa67] C. T. C. Wall, Poincaré complexes. I, Ann. of Math. (2) 86 (1967), 213-245.

[Wo74] J. A. Wolf, Spaces of constant curvature, Publish or Perish, Inc. (1974).

Department of Mathematics, UCL, Gower Street, London, WC1E 6BT, U.K.

Email address: j.k.nicholson@ucl.ac.uk 